\title{
A DYNAMIC STRUCTURAL MODEL FOR STOCK RETURN VOLATILITY AND TRADING VOLUME
}

William A. Brock

Blake D. LeBaron

Working Paper No. 4988

\section{NATIONAL BUREAU OF ECONOMIC RESEARCH 1050 Massachusetts Avenue \\ Cambridge, MA 02138 \\ January 1995}

This paper is part of NBER's research program in Asset Pricing. Any opinions expressed are those of the authors and not those of the National Bureau of Economic Research.

(c) 1994 by William A. Brock and Blake D. LeBaron. All rights reserved. Short sections of text, not to exceed two paragraphs, may be quoted without explicit permission provided that full credit, including $(\mathcal{O}$ notice, is given to the source. 


\title{
A DYNAMIC STRUCTURAL MODEL FOR STOCK RETURN VOLATILITY \\ AND TRADING VOLUME
}

\begin{abstract}
This paper seeks to develop a structural model that can let data on asset returns and trading volume speak to whether volatility autocorrelation comes from the fundamental that the trading process is pricing or, is caused by the trading process, itself. Returns and volume data argue, in the context of our model, that persistent volatility is caused by traders experimenting with different belief systems based upon past profit experience and their estimates of future profit experience.

A major theme of our paper is to introduce adaptive agents in the spirit of Sargent (1993) but have them adapt their strategies on a time scale that is slower than the time scale on which the trading process takes place. This will lead to positive autocorrelation in volatility and volume on the time scale of the trading process which generates the returns and volume data. Positive autocorrelation of volatility and volume is caused by persistence of strategy patterns that are associated with high volatility and high volume.

At a rough level, the model is able to qualitatively reproduce the following features seen in the data: (i) The autocorrelation function of a measure of volatility such as squared returns or absolute value of returns is positive with a slowly decaying tail. (ii) The autocorrelation function of a measure of trading activity such as volume or turnover is positive with a slowly decaying tail. (iii) The cross correlation function of a measure of volatility such as squared returns is approximately zero for squared returns with past and future volumes and is positive for squared returns with current volumes. (iv) Abrupt changes in prices and returns occur which are hard to attach to "news." The last feature is obtained by a version of the model where the Law of Large Numbers fails in the large economy limit.
\end{abstract}

William A. Brock

Department of Economics

University of Wisconsin, Madison

Madison, WI 53706
Blake D. LeBaron

Department of Economics

University of Wisconsin, Madison

Madison, WI 53706

and NBER 
A DYNAMIC STRUCTURAL MODEI FOR STOCK RETURN VOLATILITY AND TRADING VOLUME

by

William A. Brock and Blake D. LeBaron ${ }^{1}$

Department of Economics

The University of Wisconsin, Madison

First Draft, 7-15-93; This Draft, 12-04-94

1. Introduction

Our contribution in this paper is twofold. The first contribution is to take a step towards investigating modifications needed in asymmetric information asset pricing models so that their simulations are consistent with stylized time series facts of stock returns, volatility of stock returns, and trading volume. These facts, many of which are taken from the GARCH literature (cf. Bollerslev, Chou, and Kroner (1992), Bollerslev, Engle, and Nelson (1993)), are described in Section 3. The second contribution is to add adaptive dynamics of bellef formation in the context of asymmetric information models.

To put it another way, this paper seeks to develop a structural model that can let data on asset returns and trading volume speak to whether volatility autocorrelation comes from the fundamental that the trading process is pricing or, is caused by the trading process, itself. Returns and volume data argue, in the context of our model, that persistent volatility is caused by traders experimenting with different belief systems based upon past profit experience and their estimates of future profit experience.

A major theme of our paper is to introduce adaptive agents in the spirit of Sargent (1993) but have them adapt their strategies on a time scale that is slower than the time scale on which the trading process takes place. This will lead to positive autocorrelation in volatility and volume on the time scale of the trading process which generates the returns and volume data. Positive autocorrelation of volatility and volume is caused by persistence of strategy patterns that are associated with high volatility and high volume.

Here are some qualitative features seen in the data: (1) The autocorrelation function of a measure of volatility such as squared returns or absolute value of returns is positive with a slowly decaying tall for indices and a faster decaying tall for individual stocks. (11) The autocorrelation function of a measure of trading activity such as volume or turnover is positive with a slowly decaying tall. (11i) The cross correlation function of a measure of volatility such as squared returns is approximately zero for 
squared returns with past and future volumes and is positive for squared returns with current volumes. (iv) the autocorrelation function of returns is small for indices and is approximately zero for individual stocks. We want our model to be able to reproduce as many of these features as possible and have the potential for useful generalizations.

We shall modify the Lang, Litzenberger, Madrigal (1992), hereafter (LLM), two period competitive equilibrium model of trading volume by adding a choice of bellefs based upon past performance using those bellefs. The LLM (1992) model akes a nice platform for us to bulld upon because (1) they study both conditioning on signal alone, and conditioning on both price and signal, (11) they study both equilibrium returns and equilibrium volume, (11i) they study and test the implications for data on returns and volume which is implied by different versions of their "static" model. The last point is especially important for us because they emphasize the use of volume of trading around "event dates", 1.e., earnings announcement dates, to distinguish among four versions of the asymmetric information model. Since we are interested in asymmetric information models that explain the dynamic facts about returns, volume, and volatility that were outlined above, the LLM (1992) model makes a natural platform to launch our study. Because of the extra complexity introduced by the introduction of adaptive dynamics of belief formation, unlike LLM (1992), we shall restrict ourselves to the case of conditioning on signal alone.

We shall follow their notation as much as possible. Denote random varlables by bold face type. There is one risky asset and one risk free asset with gross return $R$. Let $\left\{v_{t}\right\}$ be an exogenously given "fundamental value" stochastic process where $v_{t}$ denotes the end of period $t$ value of the risky asset. The process $\left\{v_{t}\right\}$ will be assumed to be Independently and Identically Distributed Normal with mean $\bar{v}$ and finite variance $h \equiv \sigma_{v}{ }^{2}$ (abbreviated as $\operatorname{IIDN}\left(\vec{v}, \sigma_{v}{ }^{2}\right)$ ) unless otherwise noted. Let $p_{t}$ denote the beginning of period $t$ price of the asset.

We shall model the Impact of the trading process in attaching a price process $\left\{p_{t}\right\}$ to the fundamental process $\left\{v_{t}\right\}$, but we shall ignore, as do LLM (1992), the feedback from price into $\mathbf{v}_{t}$.

At the beginning of period $t$, given information set $I_{1 t}$ and beginning of period $t$ wealth $w_{1 t}$, trader 1 chooses $a_{1 t}$ units of risky asset, and $b_{1 t}$ units of bonds to maximize the conditional expectation of negative exponential utility of end of period $t$ terminal wealth $w_{1 t}$, to solve the problem 
(1.1) $\operatorname{Max}\left\{E\left[-\exp \left(-r w_{i t}\right)\right] \mid I_{i t}\right\}$, s.t., $p_{t} a_{i t}+b_{1 t}=w_{i t}, w_{i t}=\left(v_{t}-R p_{t}\right) a_{i t}+R w_{1 t}$.

Note that $v_{t}$ is realized at the end of period $t$, but decisions must be made on the basis of beginning of period $t$ information $I_{1 t}$. Under standard assumptions of conditional normality of $v_{t}$ given $I_{1 t}$, (cf. LLM (1992, p. 321)) this is equivalent to choosing $a_{1 t}$ to solve

(1.2) $\operatorname{Max}\left\{E\left[\left(v_{t}-R p_{t}\right) / I_{i t}\right] a_{i t}-(r / 2) a_{i t}{ }^{2} \operatorname{Var}\left[v_{t} \mid I_{i t}\right]\right.$

Here $\operatorname{Var}\left[v_{t} \mid I_{i t}\right]$ denotes the conditional variance of $v_{t}$ give the information set $I_{\text {it }}$.

The first order conditions for optimum $a_{1 t}$ yield the mean variance demand function,

$$
a_{i t}=D\left(p_{t} \mid I_{i t}\right)=E\left[\left(v_{t}-R p_{t}\right) \mid I_{1 t}\right] /\left(r \operatorname{Var}\left[v_{t} \mid I_{i t}\right]\right)
$$

Note that we use the notation "I" to denote "is defined to be". From now on we shall always work with mean variance demand functions.

The paper is organized as follows. Section one contains the introduction and some background on the kind of demand functions we shall use. Section two is the main body of this paper. It develops the model and the dynamics. It also gives a brief discussion of a simulation where traders are allowed to choose their beliefs every $T$ periods.

The model does a fair job of accounting for the contemporaneous correlation between volatility of returns and trading volume that is seen in the data. If $T$ is large enough the model does a fair job of accounting for the persistence and the shape of the autocorrelation functions of volume and volatility, but it does a poor job of accounting for the persistence of these autocorrelation functions if $T$ is small.

Section three presents empirical facts on autocorrelation functions and cross correlations of volume, volatility, and returns that we wish the model to help explain. Section three also conducts a simulation of a modification of the model where we allow differences across traders in the conditional means of the end-of-period value, $v$. This is done to explore robustness of the qualitative results to modest changes in model specification. Turn now to development of the model with adaptive dynamics of endogenous information collection. 
2. Asymmetric Information Model With Dynamics of Endogenous Signal Choice

Let all traders have access to a publically available information set $I_{t}$. There is a signal avallable each period for a cost of $C$ per period which adds more information to $I_{t}$. The mathematical definition of this signal shall be given shortly. We denote trader $1^{\prime}$ 's information state by $\omega_{1} \varepsilon\{-1,+1\}$ where "-1" denotes "do not buy signal" and "+1" denotes "buy signal." The information state is chosen every $T$ periods where $T \geq 1$.

All traders are assumed to choose their information states at the same dates $\{T, 2 T, 3 T, \ldots, N T, \ldots\}, T \geq 1$. This is called synchronous adjustment. We shall call the perlods $\{[N T-T, N T] ; N=1,2, \ldots\}$, epochs.

The information state $\omega_{1 t}$ is chosen at the beginning of epoch [NT-T,NT] and held fixed for each period $t, t=N T-T+1, \ldots, N T$. If +1 is chosen, the trader pays a cost $C$ for each period, and receives a signal $y_{1 t}=v_{t}+e_{1 t}$, where $\left\{e_{1 t}\right\}$ is $\operatorname{IIDN}\left(0, \sigma_{e}{ }^{2}\right)$ and is mutually independent across traders $i$, for all dates $t$. The signal is received at the beginning of each period $t$, before period $t$ demand is calculated. If -1 is chosen, the trader recelves no signal, i.e., $\sigma_{e}^{2}$ is infinite.

We shall use overbars to denote conditional means based on the information set $I_{t}$ which is publically available to all traders for nothing. Assume $e_{i t}$ is independent of $I_{t}$ for all $1, t$. Applying normal distribution theory as in LLM (1992), to equations (1.1)-(1.3) above we obtain,

$$
\begin{aligned}
& \bar{y}_{1 t} \equiv E\left[\left(v_{t}+e_{1 t}\right) \mid I_{t}\right]=E\left(\bar{v}_{t} \mid I_{t}\right) \equiv \bar{v}_{t}, \\
& f_{1 t} \equiv E\left(v_{t} \mid I_{t}, \omega_{1 t}=+1\right)=\bar{v}_{t}+\beta\left(y_{1 t}-\bar{y}_{1 t}\right), \beta \equiv \sigma_{v}^{2} /\left(\sigma_{v}^{2}+\sigma_{e}^{2}\right), E\left(v_{t} \mid I_{t}, \omega_{1 t}=-1\right)=\bar{v}_{t} .
\end{aligned}
$$

Here recall that $h \equiv \sigma_{v}{ }^{2} \equiv \operatorname{Var}\left[v_{t} \mid I_{t}\right]$, and $\sigma_{e}^{2}$ denotes the variance of the signal received by $i$ whlch is the same across all $i$ who purchase the signal. Furthermore $y_{1 t}$ denotes realized date $t$ value of signal $y_{1 t}$.

We shall sometimes suppress 1 , and/or $t$ to lighten notation. At each date $t$, follow Hellwig (1980) by treating the risk aversion and information state of trader $1,\left(r_{1}, w_{1}\right)$, as trader's characteristics. Follow LLM (1992) and assume risk aversions lie in a closed bounded interval bounded away from zero.

Here we will only treat the case where all the risk aversion parameters are the same so that the characteristics space is the binary space $\Omega \equiv\{-1,+1\}$. 
The straightforward large economy limit calculation of the characteristics distribution $F(\omega)$, we is given in the unabridged version of this paper (Brock and LeBaron (1993)).

We now consider equilibrium prices, returns, and volume. When there is no confusion we shall lighten notation by suppressing $\omega$ arguments, time arguments, and 1 subscripts in what follows. We shall sometimes substitute " $E_{\mu}$ " for " $S(). d \mu$ " to remind the reader of expectational properties w.r.t the characteristics measure $\mu$.

Let us calculate equilibrium at each period. Let "Lim" denote probability limit, and $\mu(d \omega)=f(\omega) d \omega$. For the special case $\omega \in\{-1,+1\}$ which is treated here, the measure $\mu$ just attaches mass $n_{-}, n_{+}, n_{-}+n_{+}=1$, to the elements $-1,+1$, respectively. This is done at each date $t$. We shall sometimes proceed with a general $\mu$ in order to indicate routes to easy generalization of the work reported here. These calculations follow LLM (1992) closely until we get to the dynamics part which starts at Equation (2.29).

Let $z_{t}$ denote outside shares (net supply of "liquidity" traders) per trader at date $t$ and, recall that $a_{i t}$ denotes demand by trader $i$ at date $t$.

We state the main assumptions here for convenience:

Assumptions: The stochastic processes $\left\{v_{t}\right\},\left\{e_{i t}\right\},\left\{z_{t}\right\}$ are IID Normal with mean vector $(\bar{v}, 0, \bar{z})$, and diagonal covariance matrix with diagonal elements, $h \equiv \sigma_{v}^{2}, \sigma_{e}^{2}(\omega), \sigma_{z}^{2}$.

Let $s(\omega) \equiv h(1-\beta(\omega)), \quad \beta(\omega) \equiv h /\left(\sigma_{e}{ }^{2}(\omega)+h\right)$. We shall see how excess returns depends upon precision of signals and the magnitude of $z_{t}$ in equilibrium. In order to obtain the equilibrium equation at each period, $t$, as $I-->\infty$, we equate the limiting supply per trader the limiting demand per trader. Suppressing $t$ to ease notation, we obtain,

$$
z=\operatorname{Lim}\left((1 / I) \sum a_{1}\right) \equiv \int a_{1} d \mu \equiv \int\left[\left(f_{1}\left(\omega_{1}\right)-R p\right) / r s\left(\omega_{1}\right)\right] d \mu=
$$

$\int\left[\bar{v}+\beta\left(\omega_{1}\right)\left(v+e_{1}-\bar{v}\right)-R p\right] / r s\left(\omega_{1}\right) d \mu \equiv n_{-}[\bar{v}-R p] / r h+n_{+}\left[\bar{v}+\beta_{+}(v-\bar{v})-R p\right] / r s_{+}$.

Note that we have dropped the subscript $t$, and the " $S$ " is w.r.t. the characteristics measure $d \mu(\omega)$. The right hand side follows from specialization of $d \mu$ to two types $\{-1,+1\}$ with $n_{-} \equiv d \mu(-1), n_{+} \equiv d \mu(+1)$. Here $s_{+}=h(1-\beta(+1)), \beta_{+} \equiv \beta(+1), \beta_{-} \equiv \beta(-1)=0$. Note that, for the signal, $\left\{0_{1}\right\}$, one can use the assumed independence of $\left\{e_{i}\right\}$ across traders 1 , to show that, 
(2.4) $\int\left[\beta\left(\omega_{1}\right) e_{1} / r s\left(\omega_{1}\right)\right] d \mu \equiv \operatorname{Lim}\left\{(1 / I) \sum\left[\beta\left(\omega_{1}\right) e_{1} / r s\left(\omega_{1}\right)\right]\right\}=0$,

by using Tchebychev's inequality.

Equilibration of demand per trader, against supply per trader, yields in the large economy limit,

(2.5a) $\left.z=\int\left(\left[\bar{v}+\beta\left(\omega_{1}\right)\left(v+e_{1}-\bar{v}\right)-R p\right)\right] / r s\left(\omega_{1}\right)\right\} d \mu=(\bar{v}-v) E_{\mu}((1-\beta) / r s)+(v-R p) E_{\mu}(1 / r s)$.

We rewrite (2.5a) for our special case below,

(2.5b) $r h z=n_{-}\left[\bar{v}-R_{p}\right]+n_{+}\left[\bar{v}+\beta_{+}(v-\bar{v})-R_{p}\right] /\left(1-\beta_{+}\right)$.

Rewrite this as follows,

(2.6a) $x=v-R p=[r z h-(\bar{v}-v)] / \bar{\gamma}=r z h / \bar{\gamma}+(v-\bar{v}) / \bar{\gamma}$, where

$\gamma \equiv 1 /(1-\beta)=1+h / \sigma_{e}^{2} \equiv 1+s i g n a l /$ noise $r a t i 0 \equiv 1+\tau, \bar{\gamma} E_{\mu} \gamma \equiv 1+\bar{\tau}, 0 \leq 1 / \bar{\gamma} \leq 1$. Denote the precision of the signal by

$\tau_{+} \equiv \mathrm{h} / \sigma_{e}^{2}(+1)$

Note that $\tau_{-}=0$ because $\sigma_{e}^{2}(-1)=\infty$. Put $\bar{\tau} \bar{m}_{-} \tau_{-}+n_{+} \tau_{+}=n_{+} \tau_{+}$and rewrite (2.5b) to obtain,

(2.6b) $\quad x=v-R p=[r z h+(v-\bar{v})] /(1+\bar{\tau}), \quad R p=(\bar{v}-r z h) /(1+\bar{\tau})+v \bar{\tau} /(1+\bar{\tau})$.

Note that $\bar{\tau} \equiv_{n_{+}} \tau_{+}$is a measure of "market precision."

Let $x=v-R p \equiv e x c e s s$ profits per share. Computing the expectation and variance of $x$ over the $v, z$ distribution, conditional on $n_{+}$, we have

(2.7a) $\operatorname{Ex}=E(v-R p)=r \bar{z} h /(1+\bar{\tau})$,

(2.7b) $\sigma_{x}^{2}=\left[r^{2} \sigma_{z}^{2} h^{2}+h\right] /(1+\bar{\tau})^{2}$,

(2.8) $\sigma_{\mathrm{Rp}}^{2}=\left[r^{2} \sigma_{z}^{2} h^{2}+h \bar{\tau}^{2}\right] /(1+\bar{\tau})^{2}$.

Note that, for the case when $\sigma_{z}^{2}=0$, an increase in $\bar{\tau}$ increases $\sigma_{\mathrm{Rp}}^{2}$ and $\bar{\tau}=\infty$ implies $\sigma_{\mathrm{Rp}}^{2}=\mathrm{h}$. Hence $\sigma_{\mathrm{Rp}}^{2}$ is always less than or equal to the fundamental 
variance, $h$, unless variance is added by outside share variance or some other force.

We wish to allow the data to speak to the presence of forces that add variance to the fundamental without "forcing" the data to push these forces into z. In order to accomplish this goal, Section three adds a group of traders that have biased conditional means. We show in Section three that this modification will allow the model to increase, rather than decrease, variance, $\sigma_{\mathrm{Rp}}^{2}$.

Turning to volume, we wish to see how volume measures relate to equilibrium returns, expected returns, and volatility of returns. In order to obtain analytical results we calculate the large economy limit of the unconditional mean of volume. Do this by using the formula for the expectation of the absolute value of a normally distributed random variable (Equation (2.19) below) and calculating as in (2.9) below.

(2.9) $\quad \hat{E V}=(1 / 2 I) \Sigma E\left|\Delta a_{1}\right|-->E V \equiv(1 / 2) \int E\left|\Delta a_{1}\right| d \mu_{2}=(1 / 2) \int E\left|\Delta\left[\left(f_{1}-R p\right) / r s\right]\right| d \mu_{2}$

$$
=(1 / 2) \int E\left|\Delta[(v-R p) / r s]+\Delta[(\bar{v}-v)(1-\beta) / r s]+\Delta\left[\beta e_{1} / r s\right]\right| d \mu_{2} \text {. }
$$

Here we denote the types measure by $\mu_{2}\left(d \omega^{\prime}, d \omega\right)=f\left(\omega^{\prime}, \omega\right) d \omega^{\prime} d \omega$ to remind the reader that one has to take into account potential dependence in the measure across two consecutive periods.

For simplicity let us assume risk aversion type does not change over time. In order to ease notation, we recall that $h \equiv \sigma_{v}{ }^{2}$, and put $\varepsilon \equiv v-\bar{v} \equiv$ "surprise." We have,

(2. 10) $2 r E V=\int E\left|\Delta\left[\left(f_{1}-R p\right) / s\right]\right| d \mu_{2}=\int E\left|\Delta\left[\left(f_{1}-R p\right) /(h(1-\beta))\right]\right| d \mu_{2}=$

$\left.\int E \mid \Delta[x /(h(1-\beta))]+\Delta[-\varepsilon / h]+\Delta\left[\beta e_{1} / h(1-\beta)\right)\right]\left|d \mu_{2}=\int E\right| \Delta A+\Delta B+\Delta C \mid d \mu_{2}$,

where,

(2. 11) $\Delta A \equiv \Delta[x /(h(1-\beta))], \Delta B=\Delta[-\varepsilon / h], \Delta C=\Delta\left[\beta e_{1} /(h(1-\beta))\right]$,

$\Delta X \equiv X^{\prime}-X$, and $X^{\prime}$ denotes next period $X$.

The equilibrium values of $\Delta A, \Delta B, \Delta C$, are given by,

(2.12a) $\Delta A=(1 / h) \Delta\{(\gamma / \bar{\gamma})(r z h+\varepsilon)\}$ 
(2. 12b) $\quad \Delta A=(1 / h) \Delta[x(1+\tau)]=(1 / h) \Delta[(1+\tau)(r z h+(v-\bar{v})) /(1+\bar{\tau})]$,

(2. 13) $\Delta B=\left\{-\varepsilon^{\prime} / h^{\prime}+\varepsilon / h\right\} \equiv \Delta\{-\varepsilon / h\}$,

(2. 14) $\Delta C=(1 / h) \Delta\left[\tau e_{1}\right]$.

The quantity, EV, can be calculated from (2.10) under normality. We wish to study the impact of an increase in precision $\tau_{+}$of the signal upon (i) volatility of excess profits per share as measured by $x_{t}^{2}$, (i1) volatility of returns as measured by volatility of $p r i c e$ change $\left|R\left(p_{t}-p_{t-1}\right)\right|$, and (iii) trading volume. We also want to lllustrate the compression effect on trading volume that occurs when fundamental variance, $h \equiv \sigma_{v}{ }^{2}$, is increased.

Consider calculation of a sample moment over [NT-T,NT], for example,

$$
M_{T} \equiv(1 / T) \sum x^{2}-T+t
$$

where $\sum$ runs over $t=1,2, \ldots, T$. Since Tchebychev's inequality implies

(2.16) $\operatorname{Pr}\left(\left|\mathrm{M}_{\mathrm{T}}-\mathrm{EM} \mathrm{M}_{\mathrm{T}}\right| \geq \varepsilon\right\} \leq \operatorname{Var}\left(\mathrm{M}_{\mathrm{T}}\right) / \varepsilon^{2}$,

and, under weak dependence of $\left\{x_{t}{ }^{2}\right\}, \operatorname{Var}\left(K_{T}\right)$ is of order $1 / T$, we may approximate the sample moment $M_{T}$ with the population moment EM for $T$ large. This type of approximation will be important for our formulation of dynamics which appears below.

Compute EV by using the general formula,

(2. 17) $2 r E V=\int\{E|\Delta A+\Delta B+\Delta C|\} d \mu_{2} \equiv \int E\left|\tilde{G}\left(\omega, \omega^{\prime}\right)\right| \mu_{2}\left(d \omega, d \omega^{\prime}\right)$, where,

(2. 18) $\tilde{G}\left(\omega, \omega^{\prime}\right)=\Delta\{(\gamma / h)(r z h / \bar{\gamma}+\varepsilon / \bar{\gamma})\}+\Delta\{-\varepsilon / h\}+\Delta\left\{\left(1 / \sigma_{e}\right) w_{1}\right\}$,

$$
=\Delta\{r z(\gamma / \bar{\gamma})\}+\Delta\{(1-\gamma / \bar{\gamma})(-\varepsilon) / h\}+\Delta\left\{\left(1 / \sigma_{e}\right) w_{1}\right\} \text {, }
$$

where conditional on $\left(\omega, \omega^{\prime}\right), \tilde{G}\left(\omega, \omega^{\prime}\right)$ is $N\left(a\left(\omega, \omega^{\prime}\right), b^{2}\left(\omega, \omega^{\prime}\right)\right)$.

Here is the formula (cf. LLM (1992)) for the expectation of the absolute value of a normally distributed variate $\tilde{G}$ which is $N\left(a, b^{2}\right)$ :

(2. 19) $E|\tilde{G}|=\left(1 /(2 \pi)^{1 / 2}\right) 2 b \exp \left[-(1 / 2)(a / b)^{2}\right]+a\{1-2 \Phi(-a / b)\}$,

where $\Phi(x)$ is the cumulative distribution function of a $N(0,1)$ variate 
evaluated at $x$.

Assume $n_{+}, n_{-}$do not change over the $T-s$ lab of time, [NT-T,NT]. Recall that $v$ and $z$ are independent and use (2.17), and (2.18) to compute EV over [NT-T, NT] to obtain,

(2.20) 2rEV=n_E\{ $|\Delta A(-1)+\Delta B(-1)+\Delta C(-1)|\}+n_{+} E\{|\Delta A(+1)+\Delta B(+1)+\Delta C(+1)|\}$

$=(2 / \pi)^{1 / 2}\left\{n_{-} b_{-}+n_{+} b_{+}\right\}$,

(2.21a) $\quad b_{-}^{2} \equiv 2\left\{r^{2} \sigma_{z}^{2}\left(\gamma_{-} / \bar{\gamma}\right)^{2}+\left(1-\gamma_{-} / \bar{\gamma}\right)^{2} / h+\tau_{-} / h\right\}$

$$
=2\left\{r^{2} \sigma_{z}^{2}[1 /(1+\bar{\tau})]^{2}+\left[\bar{\tau} /((1+\bar{\tau})]^{2} / h\right\}\right. \text {, }
$$

(2.21b) $\quad b_{+}^{2} \equiv 2\left\{r^{2} \sigma_{z}^{2}\left(\gamma_{+} / \bar{\gamma}\right)^{2}+\left(1-\gamma_{+} / \bar{\gamma}\right)^{2} / h+\tau_{+} / h\right\}$

$$
=2\left\{r^{2} \sigma_{z}^{2}\left[\left(1+\tau_{+}\right) /(1+\bar{\tau})\right]^{2}+\left[\left(\bar{\tau}-\tau_{+}\right) /(1+\bar{\tau})\right]^{2} / h+\tau_{+} / h\right\} .
$$

Note three important points that follow from (2.20) and (2.21). First, for $\sigma_{z}{ }^{2}=0$, an increase in risk aversion $r$ makes trading volume fall. Second, an increase in fundamental variance, $h$, makes trading volume fall. Third, when $\sigma_{z}{ }^{2}=0$, an increase in signal precision $\tau_{+}$makes trading volume rise, provided $n_{+}>0$. Also note that when $\sigma_{z}{ }^{2}=0$, we have $n_{-}=1$ implies $E V=0$, but $n_{+}=1$ implies positive trading volume induced by differences in individual signal realizations.

We are now at a position where we can compute and compare $\mathrm{Ex}_{\mathrm{t}}{ }^{2}, \sigma_{\mathbf{x}}{ }^{2}$, EI $p_{t}-p_{t-1}$ l, EV over the $T-s$ lab of $t$ ime [NT-T,NT] assuming no one revises beliefs over this $T-s$ lab of time. We also recall the assumption that conditional variance, $\sigma_{v}^{2}$ and conditional mean $\bar{v}$ do not change. From (2.6b) we obtain,

(2.22) $E x^{2} \equiv E(v-R p)^{2}=\left(r^{2} E z^{2} h^{2}+h\right) /\left(1+n_{+} \tau_{+}\right)^{2}$.

Note that $E x^{2}$ falls as $\tau_{+}$increases.

Perhaps the most important result of all is the finding that when fundamental variance, $h$, increases, trading volume falls. We have called this important effect, the compression effect ${ }^{2}$. It is important because it exposes a force in asymmetric information models that potentially conflicts with the well documented contemporaneous correlation between volume and volatility 
which is discussed in the empirical part of section 3 below.

Compute $\operatorname{EIR}\left(p_{t}-p_{t-1}\right)$ l over the T-slab of $t$ ime, [NT-T,NT]. Assume $z_{t}$ is constant and nonrandom over [NT-T,NT] in order to isolate out the impact of an increase in $\tau_{+}$. Take $\Delta$ of both sides of (2.6b) to obtain, using $\Delta \bar{v}=0, \Delta z=0$,

\section{(2.23) $\Delta x=\Delta v-R \Delta p=\Delta v /(1+\bar{\tau})$.}

\section{(2.24) $R \Delta p=\bar{\tau} \Delta v /(1+\bar{\tau})$.}

At this point we must pause to make a parenthetical remark. We may use (2.24) to compute the autocovariance of $\Delta \mathbf{p}^{\prime} \mathbf{x p}_{t+1}-\mathbf{p}_{t}$ with $\Delta \mathbf{p}_{\mathbf{p}} \mathbf{p}_{t}-\mathbf{p}_{t-1}$ across a string of periods where $\bar{\tau}$ does not change. One obtains, $\mathrm{R}^{2} \operatorname{Cov}\left(\Delta \mathrm{p}^{\prime}, \Delta \mathrm{p}\right)=-\mathrm{h}[\bar{\tau} /(1+\bar{\tau})]^{2}$. When $\left\{v_{\mathrm{t}}\right\}$ is $\operatorname{IIDN}(0, \mathrm{~h})$ this autocovariance is negative due to the "overdifferencing" of $v$, unless market precision $\bar{\tau}$ is zero. This is counterfactual because the tables in Section three show that the autocovariance of "simple returns" is near zero (slightly negative) for individual firms at the dally frequency. One could " $f$ ix" this by redoing our analysis by making $\left\{v_{t}\right\}$ a random walk with $\operatorname{IIDN}\left(\mu, \sigma^{2}\right)$ innovations. However, using $\Delta v=v_{t}-v_{t-1}=\mu+\eta_{t}, \eta_{t} \operatorname{IIDN}\left(0, \sigma^{2}\right)$, this leads to

(2.24') $R \Delta p=\eta_{t-1} /(1+\bar{\tau})+\left(\mu+\eta_{t}\right)(\bar{\tau} /(1+\bar{\tau})), R^{2} \operatorname{Cov}\left(\Delta p^{\prime}, \Delta p\right)=\sigma^{2}\left[\bar{\tau} /(1+\bar{\tau})^{2}\right]$

This is still counterfactual unless $\bar{\tau}$ is near zero or infinity. However, we are not too worried about this discrepancy because (i) the implication of

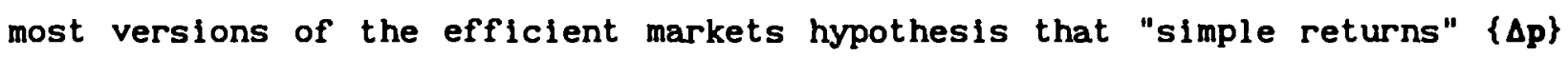
should not be autocorrelated is approximately valid only for high frequencles where effects induced by $R$ and by dividends are small; (11) our asset lives only two periods; (iil) de Fontnouvelle (1994b) extends our setup to infinite lived assets and gets better results, especially for the autocorrelation of returns. But de Fontnouvelle has to resort to approximation methods to obtain results for his more complicated model, whereas we obtain some analytical results. Hence, we shall focus on replication of time series features of volume and volatility, rather than low autocorrelation of simple returns. Return now to calculation of volatility and volume measures.

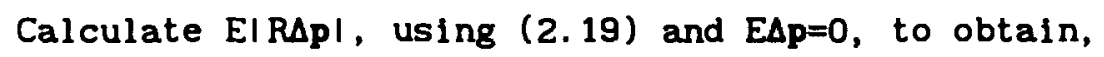

$$
E|R \Delta p|=(2 / \pi)^{1 / 2}[\bar{\tau} /(1+\bar{\tau})](2 h)^{1 / 2}
$$

It is obvious, for $n_{+}>0$, that an increase in $\tau_{+}$causes EIRApl and the 
variance of $\Delta p$ to rise. Recall that EV increases with increased $\tau_{+}{ }^{-}$Finally a similar argument as that used on $E x_{t}{ }^{2}$ shows that $\sigma_{x}{ }^{2}$ falls as $\tau_{+}$increases. We may now sum up the discussion up to this point,

Conclusion: Increased signal precision leads to increased trading volume, increased price change volatility, but decreased expected squared excess profits per share. The data described in section 3 below shows a strong contemporaneous correlation between squared returns and trading volume. Hence the model has the potential to be consistent with this strong contemporaneous correlation between squared returns and trading volume.

Recall that we have stressed the fact that the absolute value of price change $\left|p_{t}-p_{t-1}\right|$ is positively correlated with volume of trade between period t-1 and period $t$ (e.g. Gallant, Rossi, Tauchen (1993)). We have shown above that EIRApI and EV move together as $\tau_{+}$changes for a fixed level of fundamental variance.

Turn now to a brief treatment of dynamics in the choice of signal quality. Up to now we have simply followed LLM (1992) and calculated equilibrium returns, trading volume, and measures of volatility for the case where fraction $n_{\text {_ }}$ of the traders have access to a signal of precision $\tau_{-}=0$, and $n_{+}$have access to a signal of precision $\tau_{+}>0$. Now we shall introduce dynamics of $n_{-}, n_{+}$. The dynamics will be introduced by "pasting" a sequence of "static" LLM models together and "grafting" the dynamics onto this sequence.

\section{DYNAMICS}

Traders are assumed to know.the stochastic structure of the economy except for $\bar{\gamma}=1+\bar{\tau}$, which they must forecast ${ }^{3}$. We assume all risk aversion parameters $r_{1}$ equal a common value $r$. We need to calculate some expressions for input into the dynamics (2.42) below.

For type $\omega$, profits are defined by,

(2.29) $\pi(\omega) \equiv\left[\left(f_{i}(\omega)-R p\right) / r h(1-\beta(\omega))\right][v-R p]-c(\omega)$,

where $c(\omega) \equiv$ cost of purchase of signal $\omega$. All symbols in (2.29) carry the subscript $t$. Recall that $p$ is beginning of period $t$ price, $v$ is end of period $t$ value of the asset, and $x=v-R_{p}$ is excess profits per share between the beginning of period $t$ and the end of period $t$. Assume common knowledge of the 
structure of the economy and common expectations $\overline{\boldsymbol{r}}=1+\bar{\tau}$. Recall that the signal for each trader 1 is given by $y_{1}=v+e_{1}$, where $e_{1}$ is independent of $v$. Calculate expected profits (over $\left(v, z, e_{i}\right)$, suppressing " 1 " and $\omega_{1}$ arguments to lighten notation) for type $\omega$, to obtain,

(2.30) $E \pi(\omega)=E\{(\bar{v}+\beta(y-\bar{v})-R p)(v-R p)\} / r s-c(\omega)=E \pi_{0}(\omega)-c(\omega) \equiv U(\omega)$,

(2.31) $U(\omega) \equiv E\{[(v-R p)+(\bar{v}-v)(1-\beta(\omega))](v-R p)\} /[r h(1-\beta(\omega))]-c(\omega)$,

(2.32) $v-R p \equiv[r z h+(v-\bar{v})] / \bar{\gamma}$,

(2.33) $E \pi_{0}(\omega)=(1 / r h)\left\{\gamma(\omega) E\left\{(v-R p)^{2}+(\bar{v}-v)(v-R p)\right\}\right\}$

(2.34) $E \pi_{0}(\omega)=(1 / r h)\left\{\gamma(\omega)\left[r^{2} E z^{2} h^{2} / \bar{\gamma}^{e 2}+h / \bar{\gamma}^{e 2}\right]-h / \bar{\gamma}^{e}\right\}=$

$$
\left(1 / r \bar{\gamma}^{e 2}\right)\left\{\gamma(\omega) r^{2} E z^{2} h+\gamma(\omega)-\bar{\gamma}^{e}\right\} .
$$

(2.35) $\quad E \pi(\omega)=\left(1 / r \bar{\gamma}^{-2}\right)\left\{\gamma(\omega) r^{2} E z^{2} h+\gamma(\omega)-\bar{\gamma}^{e}\right\}-c(\omega)$.

(2.36) $U(\omega) \equiv E \pi(\omega)=\left(1 / \Gamma \bar{\gamma}^{e 2}\right)\left\{\gamma(\omega) r^{2} E z^{2} h+\gamma(\omega)-\bar{\gamma}^{e}\right\}-c(\omega)$.

Specialize to the case where $c(-1)=0$ :

(2.37) $U(\omega)=\left(1 / \Gamma \bar{\gamma}^{-2}\right)\left\{\gamma(\omega) r^{2} E z^{2} h+\gamma(\omega)-\bar{\gamma}^{e}\right\}-c(\omega)$.

(2.38) $d U \equiv U(1)-U(-1)=F\left(\bar{\gamma}^{e}\right) d \gamma-c(1), d \gamma=\gamma(1)-\gamma(-1)=\tau_{+}$,

(2.39) $F\left(\bar{\gamma}^{e}\right) \equiv\left(1 / r \bar{\gamma}^{e 2}\right)\left\{r^{2} E z^{2} h+1\right\}$.

We are now ready to discuss dynamics. Traders must forecast $P \equiv 1 /(1+\bar{\tau})^{2} \equiv 1 / \bar{\gamma}^{2}$. Think of $P$ as an "implicit price" for information. Every $T$ periods, traders are allowed to choose whether to buy the signal ( $\omega=1)$ or not buy the signal $(\omega=-1)^{4,5}$. At NT they have observed $T$ realizations of trading profits from trading over time periods in [NT-T,NT]. They construct the sample analogue to $E \pi(\omega)$. We assume that $T$ is large enough that the sample analogue is a good approximation to the population moment $E \pi(\omega)$. From now on we use the population moment as input to the discrete choice model below. Consider a trader 1 , whose choice probability is independent of its choice 
last epoch. Recall that $T, 2 T, 3 T, \ldots, N T, \ldots$ are called "epochs." We assume the resulting measure $\mu(d \omega)$ is formed by a discrete choice model

(2.40) $\tilde{U} \equiv U(\omega)+\iota \varepsilon(\omega)$,

where $\{\varepsilon\}$ is IID Extreme Value distributed (IIDEV) across $\omega$ in $\Omega(-1,+1\}$ as in Manski and McFadden (1981) and Anderson, de Palma, and Thisse (1993), where $\rho \equiv 1 / \ell$ is choice intensity.

The discrete choice model $(2.40)$ is attractive because it leads to tractible choice probabilities for the dynamics we develop below. Furthermore, in the dichotomous case that we examine here, the discrete choice model (2.40) allows easy extension to include interaction effects which can pile up in the large economy limit to produce discontinuous reponses to small changes in the payoffs to the cholces. This extension is briefly discussed and summarized by Equations $(2.46 a, b)$ below.

Manski and McFadden (1981) and Anderson, de Palma, and Thisse (1993) show, (note: use of " $Z$ " changes),

(2.41) $\operatorname{Pr}\{$ choose $\omega \varepsilon \Omega\} \equiv \mu(d \omega)=\exp [\rho U(\omega)] / Z, Z \equiv \sum \exp [\rho U(\nu)]$.

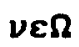

At time NT, we have, letting $\tanh (x) \equiv[\exp (x)-\exp (-x)] /[\exp (x)+\exp (-x)]$,

$$
\begin{aligned}
& m_{N T} \equiv n_{+}-n_{-}=\{\exp [\rho U(+1)]-\exp [\rho U(-1)]\} / Z=\tanh [\rho d U / 2] \\
& \left.=\tanh \left[\rho\left\{G\left(m^{e}\right)\{\gamma(1)-\gamma(-1))\right\}-c(1)\right\} / 2\right],
\end{aligned}
$$

$$
G\left(m^{e}\right) \equiv\left(1 / r \bar{\gamma}^{e 2}\right)\left\{r^{2} E z^{2} h+1\right\}
$$

$$
\bar{\gamma}^{e} \equiv\left(\left(1-m^{e}\right) / 2\right) \gamma(-1)+\left(\left(1+m^{e}\right) / 2\right) \gamma(1) \bar{z}_{\bar{\gamma}}+\left(m^{e} / 2\right) d \gamma .
$$

Equation (2.42) says that, at date NT, the choice of $m$ which will remain fixed throughout [NT, NT $+T$ ], is determined by expectation of the difference in profits from trading on the signal over trading without the signal, which in turn depends upon the point expectation, $\mathrm{m}^{\mathrm{e}}$. Equation (S.9) in Appendix 1 below is a more general formulation which includes trading profits over past epochs as well as just the last epoch.

In fact it is more intuitive to think of $P=1 / \bar{\gamma}^{2}$ as a "price" which must 
be predicted by the traders before making their choice, using the discrete choice model. Since traders are assumed to understand the structure of the model world they live in, they understand that $1 / \bar{\gamma}^{2}$ is a function of $\mathrm{g}$ iven by $(2.42)$ and $(2.43)$. Hence they must predict $m$ in order to predict $1 / \vec{\gamma}^{2}$ which is the "price" they will actually get for their information when they exploit it over the next $T$ periods of trading.

We can graph this dynamics by plotting $m_{N T}$ against $m^{e}$. Note that the RHS of (2.42) falls with $\mathrm{m}^{e}$. Assume the prediction $\mathrm{m}^{\mathrm{e}}$ is some function of $L$ past epoch values of $m$, i.e., $m^{e}=H\left(m_{N T-T}, m_{N T-2 T}, \ldots, m_{N T-L T}\right)$, such that $m=H(m, m, \ldots, m)$. That is to say, we ask that $H$ predict if the system has converged to $m$ for $L$ epochs in a row. Let us examine the simplest case $\mathrm{m}^{\mathrm{e}}=\mathrm{m}_{\mathrm{NT}-\mathrm{T}}$, which we call naive backwards dynamics.

Write Equation (2.42) in the reduced form,

$$
m_{N T}=\tanh \left[(\rho / 2) d U\left(m_{N T-T}\right)\right], d U(m)>0, m<\bar{m}, d U(m)<0, m>\bar{m}
$$

where $\bar{m}=\bar{n}_{+}-\bar{n}_{-}$, is the Grossmanstiglitz (1980) equilibrium, i.e., the breakeven level of informed traders relative to uninformed traders where the equilibrium rent on the signal is zero. I.e., dU( $\bar{m})=0$. Note that R.H.S. (2.45) converges to +1 for $m$ less than $\bar{m}$ and converges to -1 for $m$ greater than $\overline{\mathrm{m}}$.

The reader can use this property plus a graph of $\mathrm{m}_{\mathrm{NT}}$ against $\mathrm{m}_{\mathrm{NT}-\mathrm{T}}$ to show that the positive fixed point $m_{\rho}$ becomes unstable and turns into an attracting 2-cycle as $\rho$ passes through a "critical" value $\rho_{c}>0^{6,7,8}$.

Furthermore the analysis of Brock (1993) can be adapted to the setup here to produce limiting large economy dynamics of the form

(2. 46a) $m_{N T}=\tanh *\left[(\rho / 2) d U\left(m_{N T-T}\right)\right], d U(\bar{m})=0, d U(m)>0, m<\bar{m}, d U(m)<0, m>\bar{m}$,

$$
m_{N T}=\tanh \left[(\rho / 2) d U\left(m_{N T-T}\right)+\rho J m_{N T-T}\right]
$$

where $J \geq 0$ measures the degree of dependence across choices of \pm 1 of the set of traders, and $\tanh ^{*}(x)$ is the function which picks out the root of the equation

(2.47) $m=\tanh (x+\rho J m)$,

which has the same sign as does $x$, when there are two nonzero roots. There 
are two nonzero roots for $x=0$ if and only if $p J>1$.

Equation (2.46b) is an "approximation" to dynamics (2.46a) which is obtained by putting $\bar{\omega}=\mathrm{m}_{\mathrm{NT}-\mathrm{T}}$ in the probability expression in Equation (2.48) below and calculating the probability limit of $(1 / N) \Sigma \omega_{1}$ using this "approximate" probability. This notion of approximation is discussed in Brock (1993). Equation (2.46b) will be part of a system to be simulated in Section three.

We give a brief sketch of the theory that produces (2.46) here. Let $N$ now denote the number of traders and let,

(2.48) $\operatorname{Pr}\left\{\omega_{1}, \ldots, \omega_{N}\right\}=\exp \left[\rho\left(\sum\left\{U\left(\omega_{1}\right)-(J / 2)\left(\omega_{1}-\bar{\omega}\right)^{2}\right)\right\}\right] / Z$,

where the sum $\sum$ runs over 1 , and $Z$ is a normalization factor so that the probabilities given by (2.48) sum to one. Traders get utility $U\left(\omega_{1}\right)$ from their cholces $w e\{-1,+1\}$, but they dislike deviation from the mean, $\bar{\omega}$, of the community choices. It is shown in Brock (1993) that

(2. 49) $\bar{\omega}=(1 / N) \sum \omega_{1}=\Rightarrow m, N-->\infty$.

where $m$ solves (2.46a), (2.47) and "=\$" denotes convergence in distribution.

This modification of the dynamics allows Central Limit Theory for $\mathrm{J}$-parameterized dependence to produce discontinuous large changes in $\mathrm{m}_{\mathrm{NT}}$ to small changes across zero of $d U\left(m_{N T-T}\right)$ as $\rho J$ increases past unity. This is another reason why turning up the dial of rationality, i.e., increasing cholce intensity $\rho$, can lead to complicated dynamics.

Since the dynamics of $\left\{\mathrm{m}_{\mathrm{NT}}\right\}$ determines the dynamics of returns, volatility of returns and volume, the model suggests that increasing $\rho$ can lead to discontinuous changes in returns and volume/volatility bursts. Notice that a very small amount of "sociology," 1.e., very small J, can combine with high intensity of choice $\rho$ to produce a product $\rho J>1$.

It was shown by Brock (1993), that $\rho J>1$ Implies that tiny changes in sign of $\mathrm{dU}$ lead to discontinuous changes in $\mathrm{m}$ in the dynamics (2.46a). This in turn leads to discontinuous changes in volume, volatility, and returns that no econometriclan will ever be able to explain by attempting to find some "news" that is associated with the abrupt changes.

This seems consistent with studies which have documented the inability to associate changes in volatility and returns with "news." See for example, Cutler, Poterba, and Summers (1989), and Haugen, Talmor, and Torous (1991). 
While we belleve that a complete explanation of their results is far beyond such a simple model, our modeling shows how small differences in profit measures of signal purchase can lead to big differences in "market precision" $\bar{\tau}=n_{+} \tau_{+} \cdot$

B1g differences in $\bar{\tau}$ produce big differences in "local" variance (variance measured over a period of length $T$ ) of price change, and "local" trading volume as measured by EV. For example an abrupt increase in $\bar{\tau}$ causes an abrupt increase in local variance of price change, an abrupt change in price itself caused by an abrupt increase in welght on $v$ instead of $\bar{v}$ (cf. Equation (2.6b)), and an abrupt increase in local trading volume. If outside shares $z>0$, Equation (2.6a) implies that an abrupt increase in market precision causes an abrupt drop in local expected excess profits per share ${ }^{8}$. Ultimately, we hope to estimate a richer model where the data are allowed to speak to the size of parameters playing roles like $\rho$ and $J$. We now turn to a brief discussion of simulation results for the model of this Section.

Change periods are $t=20,40,60, \ldots$ No-change perlods are periods that are not multiples of 20 . We have I traders in the model where I is large but finite. Let $n(t)$ denote the fraction of informed traders at date $t$. This fraction was determined by (2.41) at the previous change period. At each date $t$, we draw $v_{t} \operatorname{IIDN}(0,1)$ and independently draw $e_{i t} \operatorname{IIDN}(0, .3)$ for each informed trader. For all dates $t, z_{t}=0, x_{t}$ is given by $(2.6 a)$, and $R p_{t}$ is given by (2.6b). If $t$ is a no-change date, volume is given by (2.9) with I equal to a large, but finite value. If $t$ is a change date, the new value of $n(t)$ is given by (2.41) and (2.9) is used to calculate volume as in (3.7b) and the surrounding exposition. Profits for each type of trader are given by (2.30) with "E" replaced by the analog estimator, "(1/I) $\Sigma$ ", are set equal to zero for all $t$. Parameter values are $h=1, \sigma_{e}{ }^{2}=.3$ for informed, $\tau_{-}=0, \tau_{+}=1 / .3$, ve $=0(+1)=8, c(-1)=0, \rho=.5, T=20$.

The results of the simulation are given in the fourth column of Tables 8-10. These Tables (and the Figures that accompany each) show how the autocorrelation and cross correlation functions of the models compare with the data. We are bothered by the negative autocorrelations at long lags in Tables 8 and 9. There is also a problem of large cross correlations in Table 10 . We leave it to future work to improve our understanding of the causes of these problems. We now turn to Section three. 


\section{Univariate Emplrical Modelling of Returns}

In this section we do two things, (1) we give a brief review of empirical "stylized" facts about stock returns and (11) we simulate a slight modification and simplification of the model treated in section 2.

Look ahead at the autocorrelations of returns, squared returns, trading volume, and the cross correlation of squared returns and trading volume shown for IBM in Tables 8-10 below. The results for IBM are typical for individual stocks. Look at Tables 2-7 below for a sample of individual stocks.

We do not present "standard errors" in most of the tables of autocorrelations and cross correlations to be presented below. This is so because (1) we only present the tables to bring out qualititive features we wish to explain and (11) we do not have a bellevable statistical model under which to bootstrap estimates of the standard errors.

We present two tables of cross correlations of a measure of trading volume with two measures of volatility of returns. These are taken from Antonlewicz (1994a, b, and personal communication). Her NYSE dataset is from the CRSP tapes and has 316 firms that trade every day of the sample (1.e., there are no zero volume days for any of these firms). The data are dally returns from $5 / 24 / 77$ to $12 / 31 / 91$. Here is the size distribution of these firms in quartiles:

Table 1: Distribution of Stock Market Values of 316 Firms ( $\$$ Mill)

Min 1st Quart Med 3rd Quart Max

$\begin{array}{lllll}73.3 & 601.7 & 1,289.9 & 2,782.1 & 41,852.3\end{array}$

In the tables below, the volume for each firm has been transformed by taking the number of shares traded each day scaled by the number of shares outstanding to get the turnover ratio. The dally turnover ratio is scaled by a 100 day backward moving average and then this ratio is logged to obtain the "volume" measure that is used in the cross-correlations.

This "detrending" is done because trading volume has grown rapidly in recent years. Our model does not speak to the general source of growth of trading volume. This general growth, we belleve, has more to do with institutional changes such as growth of modern risk management techniques, growth of mutual funds, growth of the use of derivatives and the like. We view this general growth as a "low frequency" phenomenon which trading models based upon differential information do not speak to. Hence we attempted to remove this general growth by detrending the volume series. We belleve the 
information model is designed to speak more to fluctuations around the growth trend, not to the growth trend itself.

LeBaron (1992) shows that the qualitative features of the time serles properties of the trading volume measure are falrly robust to the method of detrending--at least at the level of description that we are attempting to replicate in this paper.

The cross correlations are shown below for volume with absolute return and volume with squared return. Let $r(t)$ denote return at day $t$. The returns are calculated exactly as in the CRSP tapes. These tables show the cross-sectional distribution of the cross correlations. The reader may just use the median if she wishes. The cross correlations are reported for 5 lags on each side. As one can see, the use of $|r(t)|$ or $r^{2}(t)$ doesn't make much difference in the value of the cross correlations; except the contemporaneous correlation for $|r(t)|$ is higher than for $r^{2}(t)$. Note the positive contemporaneous cross correlation and the asymmetry of cross correlation near lag zero. In the tables, $V(s)$ denotes trading volume at time $s$. The description of the data for Tables 4 and 5 is the same as that for Tables 2 and 3 . 
Table 2. Correlation of Trading Volume $V(t-j)$ with Squared Returns $\left.r^{2}(t)\right)$.

$t$ Min 1st quart Med 3rd Quart Max

$\begin{array}{rrrrrr}-5 & -.037 & .017 & .030 & .045 & .106 \\ -4 & -.020 & .022 & .035 & .049 & .107 \\ -3 & -.015 & .032 & .047 & .064 & .132 \\ -2 & .007 & .049 & .066 & .090 & .164 \\ -1 & .028 & .091 & .123 & .164 & .279 \\ 0 & .056 & .142 & .181 & .223 & .381 \\ 1 & .007 & .058 & .074 & .092 & .171 \\ 2 & -.015 & .036 & .049 & .067 & .135 \\ 3 & -.031 & .027 & .041 & .056 & .122 \\ 4 & -.033 & .019 & .037 & .053 & .112 \\ 5 & -.040 & .013 & .030 & .045 & .106\end{array}$

Table 3. Correlation of Trading Volume $V(t-j)$ with Absolute Returns $|r(t)|$.

j Min 1st quart Med 3rd Quart Max

$\begin{array}{rrrrrr}-5 & -.051 & .016 & .034 & .051 & .109 \\ -4 & -.016 & .026 & .042 & .057 & .112 \\ -3 & -.028 & .034 & .054 & .072 & .127 \\ -2 & -.002 & .057 & .078 & .099 & .177 \\ -1 & .036 & .120 & .160 & .204 & .318 \\ 0 & .130 & .209 & .261 & .306 & .442 \\ 1 & .007 & .058 & .075 & .092 & .171 \\ 2 & -.015 & .036 & .049 & .067 & .135 \\ 3 & -.031 & .027 & .041 & .056 & .122 \\ 4 & -.034 & .019 & .037 & .053 & .112 \\ 5 & -.040 & .013 & .030 & .045 & .106\end{array}$

Data: The returns data are daily CRSP value weighted returns with dividends from $5 / 24 / 77$ to $12 / 31 / 91$. The volume series for each firm comes from the NYSE turnover ratio, which is the total shares traded divided by the number of shares outstanding. This ratio is first detrended using a 100 day moving average and then this ratio is logged to obtain the "volume" measure that is used in the cross-correlations. See Antoniewicz (1994a,b) for further properties of this data. 
Table 4. Autocorrelation Function of Squared Returns $r^{2}(t)$

Lag Min 1st Quart Median 3rd Quart Max

$\begin{array}{llllll}1 & 0.0049 & 0.1071 & 0.1498 & 0.2212 & 0.5446 \\ 2 & 0.0009 & 0.0836 & 0.1300 & 0.1963 & 0.4390 \\ 3 & 0.0003 & 0.0499 & 0.0774 & 0.1162 & 0.2777 \\ 4 & -0.0004 & 0.0347 & 0.0544 & 0.0806 & 0.2773 \\ 5 & -0.0004 & 0.0475 & 0.0744 & 0.1160 & 0.3272 \\ 6 & -0.0045 & 0.0256 & 0.0460 & 0.0731 & 0.1918 \\ 7 & -0.0048 & 0.0224 & 0.0365 & 0.0603 & 0.3484 \\ 8 & -0.0068 & 0.0232 & 0.0458 & 0.0807 & 0.2745 \\ 9 & -0.0017 & 0.0222 & 0.0426 & 0.0732 & 0.2576 \\ 10 & -0.0073 & 0.0138 & 0.0289 & 0.0508 & 0.1256 \\ 11 & -0.0043 & 0.0131 & 0.0257 & 0.0487 & 0.1453 \\ 12 & -0.0114 & 0.0128 & 0.0226 & 0.0411 & 0.1312 \\ 13 & -0.0050 & 0.0131 & 0.0271 & 0.0446 & 0.2328 \\ 14 & -0.0102 & 0.0113 & 0.0216 & 0.0404 & 0.2402 \\ 15 & -0.0108 & 0.0107 & 0.0256 & 0.0435 & 0.2622 \\ 16 & -0.0100 & 0.0077 & 0.0222 & 0.0408 & 0.2903 \\ 17 & -0.0105 & 0.0089 & 0.0193 & 0.0352 & 0.2361 \\ 18 & -0.0054 & 0.0087 & 0.0218 & 0.0388 & 0.1558 \\ 19 & -0.0123 & 0.0084 & 0.0197 & 0.0355 & 0.1166 \\ 20 & -0.0121 & 0.0073 & 0.0185 & 0.0348 & 0.1141\end{array}$

Table 5. Autocorrelation Function of Returns $r(t)$.

$\begin{array}{lcrrrc}\text { Lag } & \text { Min } & \text { 1st Quart } & \text { Median } & \text { 3rd Quart } & \text { Max } \\ 1 & -0.2831 & -0.0074 & 0.0315 & 0.0733 & 0.1750 \\ 2 & -0.0975 & -0.0413 & -0.0208 & -0.0012 & 0.0553 \\ 3 & -0.1011 & -0.0420 & -0.0270 & -0.0148 & 0.0649 \\ 4 & -0.0853 & -0.0327 & -0.0210 & -0.0058 & 0.0402 \\ 5 & -0.0714 & -0.0105 & 0.0041 & 0.0201 & 0.0750 \\ 6 & -0.0671 & -0.0163 & -0.0044 & 0.0066 & 0.0678 \\ 7 & -0.0626 & -0.0226 & -0.0087 & 0.0081 & 0.0678 \\ 8 & -0.0553 & -0.0237 & -0.0107 & 0.0016 & 0.0839 \\ 9 & -0.0599 & -0.0183 & -0.0060 & 0.0056 & 0.0540 \\ 10 & -0.0574 & -0.0153 & -0.0028 & 0.0093 & 0.0533 \\ 11 & -0.0749 & -0.0182 & -0.0056 & 0.0046 & 0.0491 \\ 12 & -0.0777 & -0.0138 & -0.0005 & 0.0125 & 0.0435 \\ 13 & -0.0515 & -0.0182 & -0.0048 & 0.0057 & 0.0457 \\ 14 & -0.0427 & -0.0107 & 0.0029 & 0.0156 & 0.0694 \\ 15 & -0.0664 & -0.0130 & 0.0010 & 0.0140 & 0.0700 \\ 16 & -0.0821 & -0.0078 & 0.0039 & 0.0152 & 0.0544 \\ 17 & -0.0502 & -0.0191 & -0.0067 & 0.0057 & 0.0597 \\ 18 & -0.0817 & -0.0144 & -0.0033 & 0.0099 & 0.0431 \\ 19 & -0.0545 & -0.0177 & -0.0032 & 0.0092 & 0.0471 \\ 20 & -0.0590 & -0.0121 & 0.0018 & 0.0160 & 0.1106\end{array}$


Note that the median autocorrelation function for these individual firms is approximately zero as one would expect from the efficient markets hypothesis. This is approximately consistent with the results of Lo and MacKinlay (1990). Lo and MacKinlay (1990, pages 180-181) show, for a sample of 4786 Individual CRSP firms, for the period July 6, 1962 to December 31, 1987, that the equal welghted index is strongly positively cross correlated, the value weighted index is positively autocorrelated and individual stocks are weakly negatively autocorrelated at dally, weekly, and monthly frequencies. They show how positive cross auto-correlations across securities are associated with positively autocorrelated index returns and weakly negatively autocorrelated individual security returns. For example, they show how the fact that returns of larger stocks lead the returns of smaller stocks in the same industry can lead to positive autocorrelation of index returns. 
Table 6. Autocorrelation Function of Trading Volume, $v(t)$

$\begin{array}{lccccc}\text { Lag } & \text { Min } & \text { 1st Quart } & \text { Median } & \text { 3rd Quart } & \text { Max } \\ 1 & 0.1751 & 0.3179 & 0.3793 & 0.4425 & 0.6072 \\ 2 & 0.1250 & 0.2284 & 0.2757 & 0.3258 & 0.5129 \\ 3 & 0.0949 & 0.1896 & 0.2287 & 0.2780 & 0.4709 \\ 4 & 0.0726 & 0.1727 & 0.2060 & 0.2533 & 0.4288 \\ 5 & 0.0662 & 0.1600 & 0.1926 & 0.2332 & 0.4123 \\ 6 & 0.0444 & 0.1357 & 0.1664 & 0.2083 & 0.3891 \\ 7 & 0.0413 & 0.1158 & 0.1513 & 0.1887 & 0.3738 \\ 8 & 0.0451 & 0.1115 & 0.1420 & 0.1758 & 0.3668 \\ 9 & 0.0351 & 0.1079 & 0.1361 & 0.1692 & 0.3504 \\ 10 & 0.0307 & 0.1075 & 0.1366 & 0.1669 & 0.3655 \\ 11 & 0.0201 & 0.0958 & 0.1215 & 0.1569 & 0.3569 \\ 12 & 0.0190 & 0.0842 & 0.1097 & 0.1452 & 0.3448 \\ 13 & 0.0045 & 0.0785 & 0.1034 & 0.1392 & 0.3488 \\ 14 & 0.0157 & 0.0780 & 0.1028 & 0.1334 & 0.3194 \\ 15 & 0.0097 & 0.0734 & 0.0992 & 0.1292 & 0.3233 \\ 16 & -0.0134 & 0.0628 & 0.0824 & 0.1105 & 0.3215 \\ 17 & -0.0068 & 0.0535 & 0.0806 & 0.1099 & 0.3081 \\ 18 & -0.0226 & 0.0545 & 0.0795 & 0.1070 & 0.3063 \\ 19 & -0.0221 & 0.0597 & 0.0809 & 0.1083 & 0.2896 \\ 20 & -0.0049 & 0.0576 & 0.0816 & 0.1044 & 0.2850\end{array}$

Table 7. Cross Correlation of Volume $v(t-j)$ with Returns $r(t)$

$\begin{array}{rcrccc}\text { Lag } & \text { Min } & \text { 1st Quart } & \text { Median } & \text { 3rd Quart } & \text { Max } \\ & & & & & \\ -5 & -0.0522 & -0.0015 & 0.0097 & 0.0255 & 0.0782 \\ -4 & -0.0414 & 0.0008 & 0.0161 & 0.0294 & 0.0854 \\ -3 & -0.0401 & -0.0019 & 0.0133 & 0.0316 & 0.0984 \\ -2 & -0.0392 & 0.0016 & 0.0170 & 0.0354 & 0.1145 \\ -1 & -0.0282 & 0.0241 & 0.0472 & 0.0762 & 0.1775 \\ 0 & -0.0156 & 0.0466 & 0.0781 & 0.1124 & 0.2292 \\ 1 & -0.0395 & 0.0034 & 0.0190 & 0.0323 & 0.0656 \\ 2 & -0.0412 & -0.0006 & 0.0093 & 0.0200 & 0.0486 \\ 3 & -0.0414 & -0.0065 & 0.0036 & 0.0135 & 0.0453 \\ 4 & -0.0297 & -0.0058 & 0.0053 & 0.0146 & 0.0506 \\ 5 & -0.0400 & -0.0072 & 0.0033 & 0.0146 & 0.0467\end{array}$


If we measure returns by $r=\left(p_{t}-p_{t-1}\right)$, then we showed in Section two that an increase in signal precision $\tau_{+}$leads to an increase in both trading volume and the variance of $\left(p_{t}-p_{t-1}\right)$. Recall that an increase in $\tau_{+}$causes equilibrium price to "soak" up some of the variance in $v$. This causes the variance of $x$ to fall. But an increase in $\tau_{+}$causes $p_{t}, p_{t-1}$ to "track" the actual realizations of $v_{t}, v_{t-1}$ better. This causes the variance of $\left(p_{t}-p_{t-1}\right)$ to rise. Since returns in many empirical studies are measured as $r_{t}=\log \left(p_{t}\right)-\log \left(p_{t-1}\right)$, a rise in the variance of $\left(p_{t}-p_{t-1}\right)$ may lead to a rise in the variance of $r_{t}$.

Sorting out whether the "soaking up" effect is stronger than or weaker than the "tracking" effect requires a model with infinitely lived assets such as de Fontnouvelle's (1994). While de Fontnouvelle gets good results with his model, we wish to stress a source of heterogeneity that leads to robust contemporaneous covariance of returns volatility with trading volume and allows the variance $\sigma_{R p}^{2}$ to be larger than the fundamental variance $h$ when the variance of $z$ is zero.

Therefore, in this section of the paper, we introduce disparity in biases of conditional means. We do not believe it is unreasonable to introduce biases of conditional means. First, as discussed by Varian (1992), this type of heterogeneity provides a way of getting around the no trade theorems reviewed by Sargent (1993). Second, Varian (1992) quotes a 1982 study by Cragg and Malkiel as saying. "We found that the best single risk measure available for each company was the extent to which different forecasters were not in agreement about that company's future growth... [These results] suggest that the variance of analysts' forecasts may represent the most effective risk proxy available." Varian quotes a source for abstracts of more recent studies that tends to support Cragg and Malkiel's conclusion.

We simulate this extended model and show that it is capable of roughly replicating features ( 1 ) and (11). In order to produce a minimalist model consistent with facts (i) and (ii) we replace the traders with access to a signal with traders whose conditional means may be biased. All traders have the same conditional variances $\sigma_{v}{ }^{2}=h$.

Put $\varepsilon=v-\bar{v}$. At period $t$ there are $n$ - potentially biased traders whose conditional means at date $t$ are given by $\bar{v}+\alpha$, and there are $n_{+}$unbiased traders whose conditional means are given by $\bar{v}$. Here $\left\{\alpha_{t}\right\}$ is an Independently and Identically Normally distributed process with mean zero and finite variance $\sigma_{\alpha}{ }^{2}$, which is independent of $\left\langle v_{t}\right\}$. Furthermore $n_{-}+n_{+}=1$. Then equilibrium returns are given, following Section 2 , 
(3.1) $x=(r h z+v-\bar{v}-\bar{\alpha})$, where

(3.2) $\bar{\alpha}=n_{-} \alpha+n_{+} 0=n_{-} \alpha=(1-m) \alpha / 2, m=n_{+}-n_{-}$.

As we said before two major issues we wish to treat with this model are: (1) How does the price formation and trading process itself contribute to inducing GARCH-type behavior of the returns even though the fundamental innovations and the bellefs themselves are IID. (11) Does GARCH-type behavior (Bollerslev, Chou, and Kroner (1992), Bollerslev, Engle, and Nelson (1993)) come through the fundamental innovations process $\left\{\varepsilon_{t}\right\}$ or come through the "structural" parameters of the model via $\bar{\alpha}$. For this article we shall define GARCH-type behavior to be a slowly decaying autocorrelation function of squared returns that is positive at all lags.

Parenthetically we remark that we want the informed traders of Section 2 in the ultimate model in order to account for anticipation effects before a release of a packet of information such as an earnings report that is partially known to informed (insider) traders before the rest. Anticipation effects exist for earnings reports as documented by, for example, Rendleman, Jones, and Latane' (1982) who show that the market "leaks" the Information into returns a few days before the release, jumps at the release, and does not fully adjust until up to ninety days after the release for the biggest earnings report "surprises."

But in this section of the paper we are just going to simulate the highly specialized version of the general model, (3.1), (3.2), but where the time scale of bellef adjustment is slower than the time scale of trading. We simulate another specialized model where the time scales are the same but where traders display more diversity of bellefs in our unabridged version, Brock and LeBaron (1993).

Lighten the notation by putting $h=\sigma_{v}^{2}, n_{-}=n, \alpha_{-}=\alpha$. Then (3.1), (3.2) and calculations of profits, strengths, etc., are summarized in Equations (3.3)-(3.6) below,

(3.3) $x_{t+1}=-n_{t} \alpha_{t}+\varepsilon_{t+1} \equiv F\left(I_{t}, \theta\right)+G\left(I_{t}, \theta\right) \varepsilon_{t+1}$, where,

(3.4a) $\quad m_{t}=\tanh \left((p / 2) \Delta S_{t}\right), \Delta S_{t} \equiv S_{+, t}-S_{-, t}$

(3.4b) $m_{t}=\tanh \left((\rho / 2) \Delta S_{t}+\rho J m_{t-1}\right)$ 
(3.5a)

$$
\pi_{+, t} \equiv-x_{t} n_{t-1} \alpha_{t-1} / r h_{t-1}, \pi_{-, t}=x_{t} \alpha_{t-1}\left(1-n_{t-1}\right) / r h_{t-1}
$$

(3.5b) $E_{t-1}\left(\pi_{+, t}-\pi_{-, t}\right)=n_{t-1} \alpha_{t-1}^{2} / r h_{t-1}$,

(3.6a) $1-n_{t}=\left(1+m_{t}\right) / 2, n_{t}=\left(1-m_{t}\right) / 2$,

(3.6b) $\quad \Delta S_{t}=\left[E_{t-1}\left(\pi_{+, t}-\pi, t\right)-C\right]+\eta \Delta S_{t-1}$.

The timing and notation convention is as follows. Differential "strength", $\Delta S_{t}$, depends on a random variable, $\alpha_{t-1}$ which was drawn before $x_{t}$ was drawn. After $x_{t}$ is realized a new "belief" $\alpha_{t}$ is drawn before $x_{t+1}$ is realized. The innovation $\varepsilon_{t+1}$ has a $t+1$ subscript because it is drawn after $\alpha_{t}$ is realized. Here $\pi_{+, t}, \pi_{-, t}$ denote profits of unblased and blased traders. Strengths are given in $(3.6 \mathrm{~b})$ where $\mathrm{C}>0$ is the cost to receive an unbiased conditional expectation $\bar{v}$ of the end of period value $v$. Equation (3.4b) is the analogue to Equation (2.46b) for this model.

Twice trading volume is given by the sum of the absolute values of the change in equilibrium demands across two consecutive periods. In order to ease notation in what follows, put $v_{t}=v, m=m_{t}, \alpha=\alpha_{t}, n=n_{t}=(1-m) / 2, S_{t}=S$ and use the convention of dropping time subscripts for current period variables and using, to denote next period variables.

In this tidied up notation, the bivariate returns and volume process is given by,

(3.7a) $x^{\prime}=-n \alpha+\varepsilon^{\prime}, \quad n=(1-m) / 2, m=\tanh [(\rho / 2) \Delta S], \Delta S^{\prime}=n \alpha^{2} / r h-C+n \Delta S$,

(3.7b) $2 \Gamma \mathrm{V}=\mathrm{n}(+1,+1)\left|\left(-\bar{\alpha}^{\prime}\right) / \mathrm{h}^{\prime}-(-\bar{\alpha}) / \mathrm{h}\right|+\mathrm{n}(-1,+1)\left|\left(\alpha^{\prime}-\bar{\alpha}^{\prime}\right) / \mathrm{h}^{\prime}-(-\bar{\alpha}) / \mathrm{h}\right|$

$+n(+1,-1)\left|\left(-\bar{\alpha}^{\prime}\right) / h^{\prime}-(\alpha-\bar{\alpha}) / h\right|+n(-1,-1)\left|\left(\alpha^{\prime}-\bar{\alpha}^{\prime}\right) / h^{\prime}-(\alpha-\bar{\alpha}) / h\right|$,

where $\bar{\alpha}^{\prime}=n^{\prime} \alpha^{\prime}, \bar{\alpha}=n \alpha, n\left(\omega^{\prime}, \omega\right)=\operatorname{Pr}\left\{\omega^{\prime}, \omega\right\}, \omega, \omega^{\prime} \varepsilon \Omega=\{-1,+1\}$.

Here, the volume equation is obtained by taking the number of traders to infinity in the definition, $2 V=(1 / N) \sum\left|\Delta D_{1}(p)\right|$ where the demands are evaluated at equilibrium $p$. Here for any bellef $\alpha_{1}=\alpha_{-}$or $\alpha_{+} \equiv 0, D_{1}(p)=\left(\bar{v}+\alpha_{1}-R p\right) / r h$. For $z=0$, we have $D_{1}(p)=\left(\alpha_{1}-\bar{\alpha}\right) / r h$, in equilibrium. A similar computation gives the equilibrium values for the other terms in the volume expression. Furthermore, for change periods, $n\left(\omega^{\prime}, \omega\right)=\operatorname{Pr}\left\{\omega^{\prime}, \omega\right\} \equiv n\left(\omega^{\prime}\right) n(\omega)$, where $n\left(\omega^{\prime}\right), n(\omega)$ are given by the choice model (3.4) across the two consecutive periods. To put it another 
way, we compute belief state probabilities using the cholce model (3.4) under the assumption of independence. Computation of $n\left(\omega^{\prime}, \omega\right)$ for no-change periods will be explained below.

Equations (3.7) give some understanding of the forces that act to create GARCH-type volatility persistence in returns, $\left\{\mathbf{r}_{t}\right\}$. We did a computer search for parameter combinations consistent with GARCH behavior for returns under the assumption that $\left\{\varepsilon_{t}\right\}$ and $\left\{\alpha_{t}\right\}$ are mutually independent, IID with mean zero and finite variance.

Under IID bellefs and IID $\left\{\varepsilon_{t}\right\}$ this search falled to find parameter values that generated equilibrium returns and volume that were consistent with the correlation pattern in the data. The squared returns and volume were not persistent enough to be consistent with the data. Some kind of mechanism to induce temporal correlations in belief heterogeneity seems to be necessary. We belleve part of the trouble is due to too much flipping in belief states between periods. Following the spirit of Section 2, we introduce a "slow" time scale where changes in beliefs take place on average once every $T$ periods. Let us explain.

There are two types of time period pairs. Time period pair $(t-1, t)$ is a change period when, $\ldots=m_{t-2}=m_{t-1}, m_{t}=\tanh \left[(\rho / 2) \Delta S_{t}\right], m_{t+1}=m_{t}, m_{t+2}=m_{t}=\ldots$, until the next change period. All other periods $(t-1, t)$ are no change periods. Change perlods are drawn Independently and Identically Distributed (IID) with a small probability in what follows.

In the simulations reported below, change times, " $t$ ", are drawn IID over time with the probability of a change time set equal to .05. This gives an average life of a no change period of twenty periods. While the qualitative features of the simulation results are robust it is important that the average life of a no change perlod be longer than the time scale of trading. This captures the idea that the time scale of adaptation of the traders is slower than the "ticker tape."

In order to see how a mechanism like this could generate persistence in $x_{t}{ }^{2}$ let $\left\{e_{t}\right\}$ be $\operatorname{IIDN}(0,1)$, let standard deviation $\sigma$ follow a two state Markov process with transition probabilities $p(j \mid i)=\operatorname{Pr}$ (go from $i$ to $j$ in one time step\}. Consider the stochastic process

(3.8) $x_{t}=\sigma_{1} e_{t}\left(x_{t}=\sigma_{2} e_{t}\right)$ with probability $p(1 \mid 1)(p(2 \mid 1)), 1=1,2$

Suppose that $\sigma_{1}=1, \sigma_{2}=3, p(2 \mid 1)=p(1 \mid 2)=.05, p(1 \mid 1)=p(2 \mid 2)=.95$. Then it is easy to check that the stationary probabllities $p(1)=p(2)=.5, E x_{t}^{2}=E x_{t+1}^{2}=5$, 
$E x_{t}^{2} x_{t+1}^{2}=.5[p(1 \mid 1)+9 p(2 \mid 1)+p(1 \mid 2)+81 p(2 \mid 2)]=.5[.95+.45+.05+76.95]=39.2>25$, so $\operatorname{Cov}\left(x_{t}^{2}, x_{t+1}^{2}\right)>0$. Note how the large probability of staying in any state once you are in that state contributes to positivity of $\operatorname{Cov}\left(x_{t}^{2}, x_{t+1}^{2}\right)$. Incidentally the autocorrelation function of the squares of a sample drawn from the process (3.8) was positive with a slow decay in the lags.

Introducing a slow time scale is rather like increasing the probability of staying in a state once you are in that state. We shall see shortly how slowing down the time scale upon which beliefs change will produce autocorrelations of $\left\{x^{2}\right\}$ and $\{T\}$ that resemble the data even though $\{\alpha\},\{\varepsilon\}$ are IID. Before we do that turn to another empirical issue.

Empirical evidence is strong that $\left|R\left(p^{\prime}-p\right)\right|$ is positively associated with v. Let us examine the ability of (3.7) to replicate this feature of the data. For $\left\{v_{t}\right\}$ IID, $\bar{v}$ is constant so $R p=\bar{v}+n \alpha$ implies

$$
\sigma_{p} \equiv\left|R\left(p^{\prime}-p\right)\right|=|\Delta(n \alpha)|
$$

We shall see in a moment that a term like (3.9) will appear in a special version of the volume formula. Look at a no change period $(t-1, t)$. Here $n\left(\omega^{\prime}, \omega\right)=\operatorname{Pr}\left\{\omega^{\prime}, \omega\right\}=\operatorname{Pr}\left\{\omega^{\prime} \mid \omega\right\} \operatorname{Pr}\{\omega\}=0$ if $\omega^{\prime}$ not equal to $\omega$ and equals $\operatorname{Pr}\{\omega\}$ otherwise. Then volume over the no change period where $n^{\prime}=n$ is given by

(3. 10) $2 \Gamma V=n(+1)\left|\left(-n^{\prime} \alpha^{\prime}\right) / h^{\prime}-(-n \alpha) / h\right|+n(-1)\left|\left(\alpha^{\prime}-n^{\prime} \alpha^{\prime}\right) / h^{\prime}-(\alpha-n \alpha) / h\right|$.

For the case $\{\varepsilon\}$ IID, $h^{\prime}=h$, we have, noting that $m=n(+1)-n(-1), m^{\prime}=m$, $n(+1) \equiv 1-n, \quad n \equiv n(-1), n^{\prime}=n$,

(3.11) $2 \Gamma V=(1 / 2 h)\left\{\left(1-m^{2}\right)\left|\alpha^{\prime}-\alpha\right|\right\}$.

Hence, $2 \Gamma V$ is proportional to $\sigma_{p} \equiv\left|R\left(p^{\prime}-p\right)\right|=|\Delta(n \alpha)|=n\left|\alpha^{\prime}-\alpha\right|$ for no change periods.

Thus we see for the special case of a no change period, the volatility measure given in (3.9) is positively related to the volume measure given by (3.11). The case of change periods is much more complicated. But, since change periods will be infrequent in the computer simulations to follow, we have done enough to suggest that volume and volatility can be contemporaneously correlated in this kind of model. 
SOME CALIBRATION RESULTS

We wish to see how well the model does in matching moments of actual dally CRSP value welghted index returns from July 3, 1962 through September 30, 1987. Take a look at the Tables with parallel Figures below 
Table 8. Autocorrelations of Squared Returns

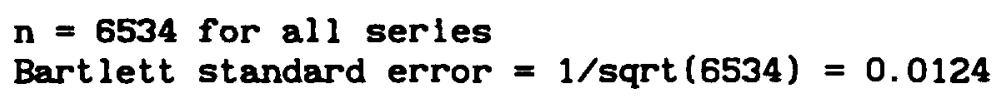

$\begin{array}{rrrrr}1 & 0.184 & 0.208 & 0.198 & 0.170 \\ 2 & 0.169 & 0.102 & 0.190 & 0.167 \\ 3 & 0.159 & 0.041 & 0.157 & 0.135 \\ 4 & 0.175 & 0.043 & 0.184 & 0.151 \\ 5 & 0.173 & 0.097 & 0.110 & 0.101 \\ 6 & 0.105 & 0.068 & 0.123 & 0.064 \\ 7 & 0.154 & 0.013 & 0.100 & 0.030 \\ 8 & 0.128 & 0.021 & 0.107 & 0.075 \\ 9 & 0.131 & 0.029 & 0.072 & 0.031 \\ 10 & 0.134 & 0.021 & 0.044 & 0.003 \\ 11 & 0.135 & 0.026 & 0.069 & 0.011 \\ 12 & 0.136 & 0.026 & 0.040 & -0.027 \\ 13 & 0.124 & 0.030 & 0.046 & -0.011 \\ 14 & 0.150 & 0.037 & 0.046 & -0.035 \\ 15 & 0.125 & 0.018 & 0.031 & -0.049 \\ 16 & 0.117 & 0.011 & 0.041 & -0.074 \\ 17 & 0.131 & 0.023 & 0.038 & -0.097 \\ 18 & 0.129 & 0.021 & 0.026 & -0.091 \\ 19 & 0.110 & 0.027 & 0.004 & -0.102 \\ 20 & 0.124 & 0.034 & 0.000 & -0.112\end{array}$
(1) = Value Weighted Index
(2) $=$ IBM
(3) = Simulated Returns of model in Section 3
(4) = Simulated Returns of model in Section 2 


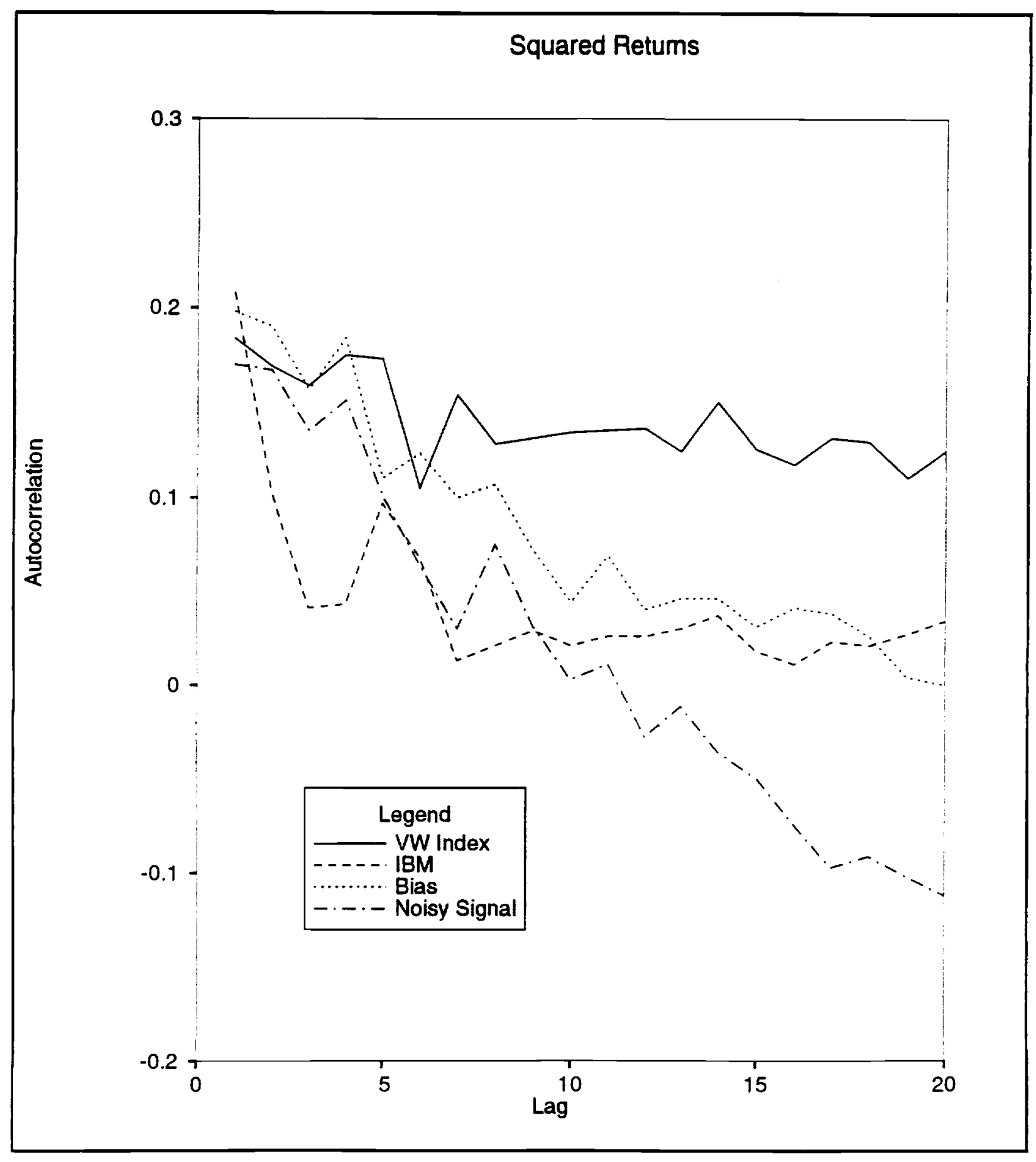


Table 9. Autocorrelations of Trading Volume

$\begin{array}{rrrrr}\text { Lag } & (1) & (2) & (3) & (4) \\ 1 & 0.690 & 0.594 & 0.296 & 0.477 \\ 2 & 0.544 & 0.435 & 0.206 & 0.367 \\ 3 & 0.493 & 0.383 & 0.201 & 0.335 \\ 4 & 0.462 & 0.351 & 0.184 & 0.303 \\ 5 & 0.430 & 0.331 & 0.176 & 0.269 \\ 6 & 0.389 & 0.279 & 0.155 & 0.229 \\ 7 & 0.366 & 0.254 & 0.166 & 0.182 \\ 8 & 0.356 & 0.232 & 0.163 & 0.160 \\ 9 & 0.324 & 0.223 & 0.152 & 0.126 \\ 10 & 0.313 & 0.210 & 0.155 & 0.080 \\ 11 & 0.296 & 0.189 & 0.153 & 0.045 \\ 12 & 0.291 & 0.164 & 0.153 & 0.015 \\ 13 & 0.267 & 0.156 & 0.174 & -0.031 \\ 14 & 0.252 & 0.151 & 0.155 & -0.059 \\ 15 & 0.239 & 0.161 & 0.154 & -0.087 \\ 16 & 0.231 & 0.138 & 0.154 & -0.132 \\ 17 & 0.230 & 0.141 & 0.114 & -0.176 \\ 18 & 0.216 & 0.134 & 0.120 & -0.212 \\ 19 & 0.211 & 0.160 & 0.119 & -0.233 \\ 20 & 0.206 & 0.169 & 0.145 & -0.211\end{array}$
(1) = Value Welghted Index
(2) $=$ IBM
(3) = Simulated Returns of model in Section 3
(4) = Simulated Returns of model in Section 2 


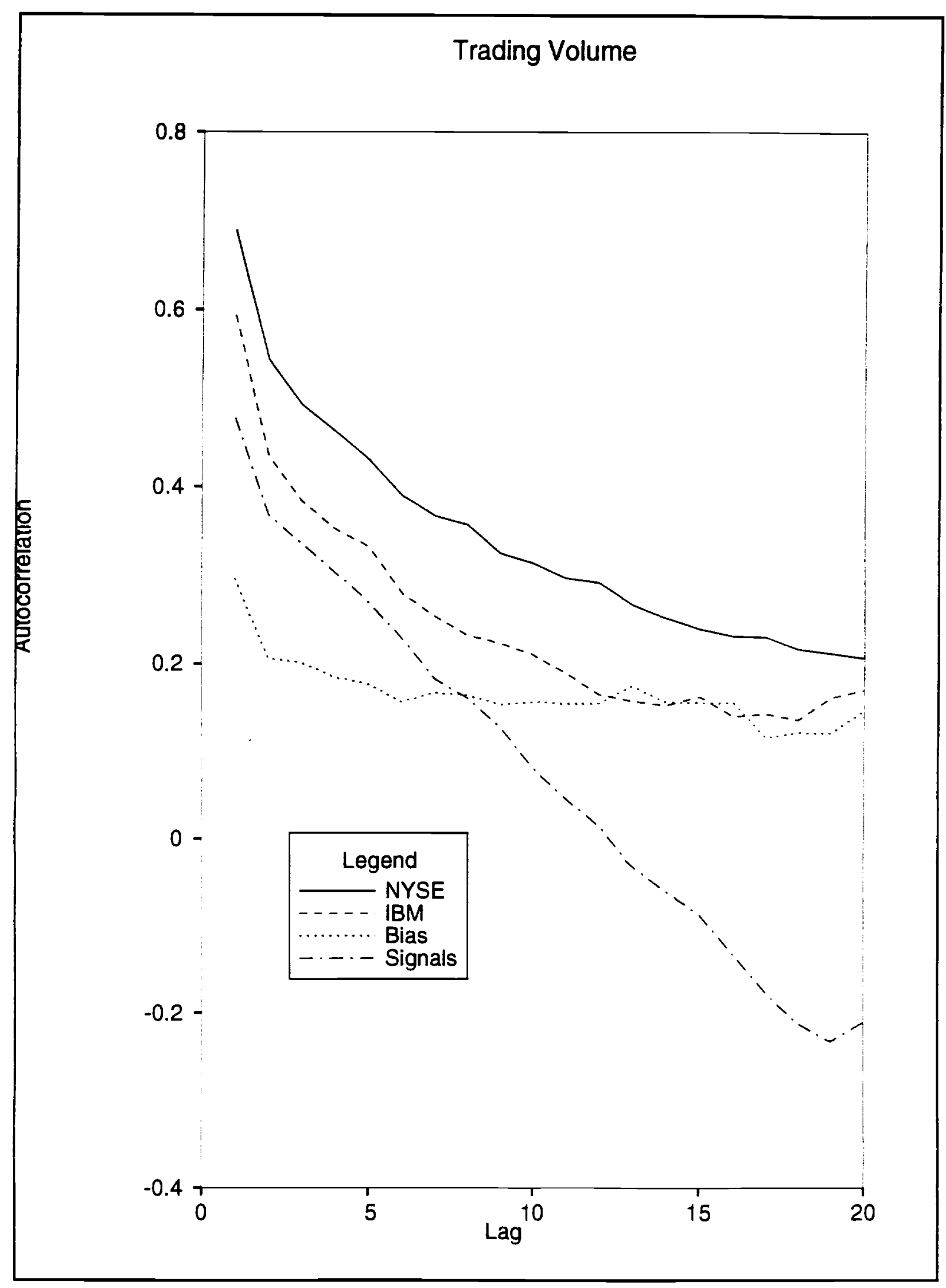


Table 10. Cross Correlation of Squared returns( $t)$ with Trading Volume $V(t+j)$.

$\begin{array}{rrrrl}\text { Lag } & (1) & (2) & (3) & (4) \\ -10 & 0.004 & 0.001 & -0.019 & 0.000 \\ -9 & -0.006 & 0.008 & -0.015 & 0.020 \\ -8 & -0.003 & 0.009 & -0.024 & 0.064 \\ -7 & -0.009 & 0.012 & -0.007 & 0.060 \\ -6 & -0.011 & 0.015 & -0.020 & 0.098 \\ -5 & -0.019 & 0.022 & 0.006 & 0.130 \\ -4 & 0.003 & 0.036 & 0.020 & 0.164 \\ -3 & 0.006 & 0.048 & 0.026 & 0.185 \\ -2 & 0.011 & 0.067 & 0.008 & 0.193 \\ -1 & 0.009 & 0.098 & 0.147 & 0.209 \\ 0 & 0.212 & 0.137 & 0.168 & 0.414 \\ 1 & 0.221 & 0.126 & 0.035 & 0.475 \\ 2 & 0.043 & 0.069 & 0.032 & 0.245 \\ 3 & 0.029 & 0.026 & 0.003 & 0.226 \\ 4 & 0.032 & 0.008 & 0.000 & 0.201 \\ 5 & 0.024 & 0.011 & -0.024 & 0.207 \\ 6 & 0.020 & 0.007 & -0.024 & 0.155 \\ 7 & 0.009 & 0.007 & -0.012 & 0.133 \\ 8 & 0.008 & -0.001 & -0.024 & 0.097 \\ 9 & 0.004 & -0.004 & -0.033 & 0.107 \\ 10 & -0.023 & -0.011 & -0.040 & 0.063\end{array}$

(1) = Value Weighted Index

(2) $=$ IBM

(3) = Simulated Returns of model in Section 3

(4) = Simulated Returns of model in Section 2

Parameter values of model in Section 3: $\rho=0.1, J=1, \quad \eta=0.75$, $\operatorname{var}($ bellefs) $/ \operatorname{var}($ fundamental) $=40$, risk aversion=1, cost of signal=8, Probability of re-evaluation period $=0.05$.

Parameter values of model in Section $2: h=1, \sigma_{e}{ }^{2}(+1)=.3, \bar{v}=0, \rho=.5, c(+1)=8$, $c(-1)=0, T=20, \tau_{+}=1 / .3$, risk aversion $=1$.

Data: The returns data are daily CRSP value weighted returns with dividends from July 3rd, 1962 through September 30th, 1987. The volume series comes from the NYSE turnover ratio, the total shares traded divided by the number of shares outstanding. This ratio is first detrended using a 100 day moving average, and then it is log transformed. Finally, day of the week effects are removed from this series giving the final volume series used here. See LeBaron (1992) for further properties of this series. 


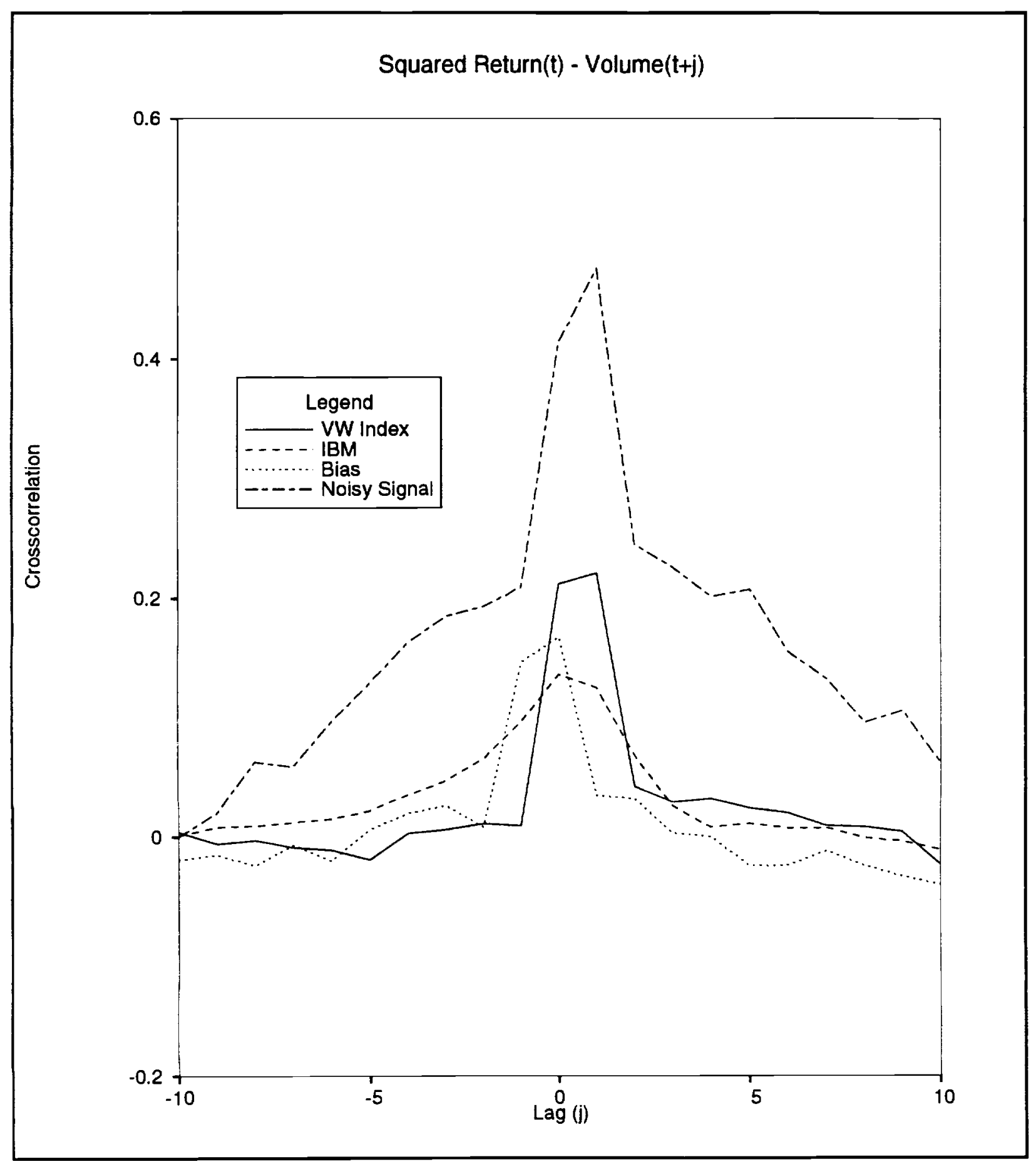


Let us discuss the Tables and Figures. Before we begin note that our model is a very severe abstraction of diversity of beliefs. But it is good enough to give the martingale difference sequence property of returns observed in the data.

The martingale difference property requires $E_{t} x_{t+1}=0$. To see why this is true, recall that, $n_{t}=\left(1-m_{t}\right) / 2, m_{t}=\tanh \left((\rho / 2)\left(n_{t-1} \alpha_{t-1}^{2} / r h_{t-1}+\eta \Delta S_{t-1}\right)\right]$. Hence if $E\left(\alpha_{t} \mid \alpha_{t-1}, h_{t-1}, \Delta S_{t-1}, \ldots\right)=0$, which we assume, we have $E_{t} x_{t+1}=-E_{t} E_{t-1} n_{t} \alpha_{t}=0$.

First, note that Table 8 shows that the autocorrelation function of squared index returns (IBM individual stock returns) is positive and Table 8 indicates that it dies slower than exponential, (dies exponentially) whereas the autocorrelation function of squared returns generated by our model is positive and dies exponentially fast. The Antoniewicz (1994a,b) findings indicate that this pattern of faster decay is a general property of individual stock returns compared to the value weighted index returns. Compare the median of Table 4 to the value weighted index of Table 8 . Notice the faster decay of the median individual autocorrelation function of squared returns.

Hence, at a crude level, we match the qualitative features of the autocorrelation function of squared returns but not the quantititive features.

Second, Table 9 shows the same type of conclusion for autocorrelations of trading volume but we do worse on the magnitude. However, note that our simulated volume autocorrelation decays slowly at large lags. This is a feature that is consistent with the data.

Third, Table 10 shows the correlation pattern between volume and volatility is qualitatively matched by our model.

We believe the model has performed well enough in matching moments to give it enough credibility to make the point that a robust channel for heteroskedasticity to enter returns is through the trading process itself and not solely through the fundamental which the trading process is attempting to price. The mechanism stressed in this section is adaptation of trading strategies on a slower time scale than the scale on which trading takes place.

SUMMARY, CONCLUSIONS, FUTURE RESEARCH

The minimalist version of our model does fairly well at replicating qualitative features of volatility and volume in the data provided belief adaptation takes place on a slower time scale than the time scale of the 
"ticker tape" or the time scale of collection of data on returns. The approach taken in this paper has serious problems, however.

First, some mechanism must be present to make large enough masses of traders to change to similar enough strategies to prevent the cross sectional law of large numbers from "washing out" the aggregate effects of such strategy diversity. While the joint probability structures of Brock (1993) which were sketched in Equation (2.48) and surrounding text present one way of allowing data to speak to the presence of clumping effects, we are still missing a microfoundation for such clumping effects.

This same criticism applies to well known important works such as DeLong, Shleifer, Summers, and Waldmann (1990) and the literature on "noise" traders, sunspots, etc. Perhaps adaptation of "lattice" based methods such as Durlauf (1993), Brock (1993), Kiefer, Ye, and An (1993) can give us a microfoundation for aggregative effects that survive the cross sectional law of large numbers. We do not apologize for this gap in our paper, but it must be pointed out.

Second, there is very little diversity in the model treated in Section three. Traders simply have the cholce of whether to pay a fee to get an unbiased predictor of $v_{t}$ or not pay the fee and get a biased predictor of $v_{t}$. Common sense would suggest that a trader that has invested in an unbiased predictor in the past would find it cheaper to acquire an unbiased predictor later. Such dependence in cost upon past information purchases is not treated here. This and other sources of diversity must be left for future research.

Third, we have treated only one type of diversity here. In our unabridged version Brock and LeBaron (1993) we treated another type of model where each bellef type had a countertype whose "biases" were opposite in sign. Simulations suggest models of this type do not generate enough volatility and volume persistence to be consistent with the data if fundamentals and beliefs are IID. But models of this type strongly suggest that GARCH fundamental processes are inconsistent with the strong contemporaneous correlation between volume and volatility seen in the data. The mechanism is simple. GARCH fundamentals lead to small mean variance demands which lead to timid trading which leads to small volume when volatility of returns is large which is counterfactual.

Fourth, while information-based models of the type treated in Section two can generate heteroskedastic returns from IID fundamentals, they generate returns with smaller variance than the fundamentals and equilibrium volume gets damped by large fundamental variance. This caused us to move to a model like that treated in Section three. However, information based models can 
generate the anticipation effects in returns before big "news" events. A nice extension would be to build models that include both components from Sections two and three and calibrate them on empirical data.

Fifth, de Fontnouvelle (1994) has extended our model to the case of infinitely lived assets. His model does a good job of matching the autocorrelation and cross correlation patterns of the data, even without the device of two time scales. The long life of the asset creates a pathway for persistence. A nice extension of both our models and de Fontnouvelle's model would be to explore dynamic impulse response functions of the models and compare these to their empirical counterparts in Gallant, Rossi, and Tauchen (1993).

Sixth, and finally, we used a combination of analysis interacting with computer simulations to produce our conclusions. We need more analytic results to understand the forces that lead to GARCH in returns. By conducting computer simulations we argued, in the context of our model, that volatility persistence enters returns through a slower time scale of changes in beliefs which enter the demand function. This time scale is slow relative to the time scale of trading. Based on our simulations this channel appears to be consistent with volatility and volume correlation patterns documented in the data.

In the context of a model where the time scales of demand change and trading are the same but where demand diversity is stronger we argued in Brock and LeBaron (1993) that volatility persistence of returns must be coming through beliefs rather than the fundamentals that the trading process is pricing. This is so because fundamental volatility peristence dampens demands of risk averse traders too much to be consistent with the cross correlation pattern of volatility and volume.

The main conclusion is this. Combined analysis and simulation of structural models of trader diversity can help us identify likely causes of volatility and volume persistence which is seen in the data. In particular it can shed light on a fundamental question: Does volatility persistence come from the fundamental that the trading process is pricing or does it come from the trading process itself? The analysis here shows how the trading process can contribute to volatility magnification (and contraction) as well as volatility persistence and persistence in trading volume. 
Anderson, S., de Palma, A., Thisse, J., (1993), DISCRETE CHOICE THEORY OF PRODUCT DIFFERENTIATION, Cambridge, Mass.: MIT Press.

Anderson, B., Moore, J., (1979), OPTIMAL FILTERING, Prentice-Hall, Inc.: Englewood $\mathrm{Cl}$ iffs, NJ.

Antoniewicz, R., (1992a), "A Causal Relationship Between Stock Returns and Volume," Federal Reserve Board, Washington, D.C.

Antoniewicz, R., (1992b), "Relative Volume and Subsequent Stock Price Movements," Federal Reserve Board, Washingrton, D.C.

Arthur, W., (1992), "On Learning and Adaptation in the Economy," Food Research Institute, Stanford University.

Arthur, W., Holland, J., LeBaron, B., Palmer, R., Tayler, P., (1993), "Artificial Economic Life: A Simple Model of a Stockmarket," Santa Fe Institute Working Paper.

Basawa, I, Prakasa Rao, B., (1980), STATISTICAL INFERENCE FOR STOCHASTIC PROCESSES, New York: Academic Press.

Black, F., (1986), "Noise," JOURNAL OF FINANCE, 41, July: 529-43.

Bollerslev, T., Chou, R., Kroner, K., (1992), "ARCH Modelling in Finance: A Review of the Theory and Empirical Evidence," JOURNAL OF ECONOMETRICS, 52, 5-59.

Bollerslev, T., Engle, R., Nelson, D., (1993), "ARCH Models," HANDBOOK OF ECONOMETRICS, VOLUME IV, Amsterdam: North Holland (forthcoming).

Brock, W., (1993), "Pathways to Randomness in the Economy: Emergent Nonlinearity and Chaos in Economics and Finance," ESTUDIOS ECONOMICOS, Vol. 8, No. 1, January-June. Also Santa Fe Institute Working Paper, \#93-02-006 and SSRI R.P. \#410, Department of Economics, University of Wisconsin, Madison.

Brock, W., Hommes, C., (1994), "Rational Routes to Randomness: Rationality in an Unstable Market with Information Costs Implies Chaos," Department of Economics, University of Wisconsin, Madison.

Brock, W., LeBaron, B., (1993), "Using Structural Modeling in Building Statistical Models of Volatility and Volume of Stock Market Returns: Unabridged Version," Social Science Research Institute Working Paper, Department of Economics, The University of Wisconsin, Madison.

Campbell, J., Grossman, S., Wang, J., (1993), "Trading Volume and Serial Correlation in Stock Returns," QUARTERLY JOURNAL OF ECONOMICS, 108, \#4, 905-939.

Cutler, D., Poterba, J., Summers, L., (1989), "What Moves Stock Prices?" JOURNAL OF PORTFOLIO MANAGEMENT, 15, Spring, 4-12.

de Fontnouvelle, P., (1994a), "Testing the Mixture of Distributions Model," Department of Economics, The University of Wisconsin, Madison. 
de Fontnouvelle, P., (1994b), "Stock Returns and Trading Volume: A Structural Explanation of the Empirical Regularities," Department of Economics, The University of Wisconsin, Madison.

De Long, J., Shlelfer, A., Summers, L., Waldmann, R., (1990), "Noise Trader Risk in Financial Markets," JOURNAL OF POLITICAL ECONOMY, 98, \#4, 703-738.

Durlauf, S., (1993), "Nonergodic Economic Growth," REVIEW OF ECONOMIC STUDIES, Vol. 60 (2), \#203, 349-366.

Easley, D., Klefer, N., O'Hara, M., (1992)., "One Day in the Life of a Very Common Stock," Center for Analytic Economics, Cornell University, 402, Uris Hal 1, Ithaca, NY., 14853-7601.

Friedman, D., Rust, J., (1993), THE DOUBLE AUCTION MARKET: INSTITUTIONS, THEORIES, AND EVIDENCE, Redwood City, California: Addison-Wesley.

French, K., and Roll, R., (1986), "Stock Return Varlances, the Arrival of Information, and the Reaction of Traders," JOURNAL OF FINANCIAL ECONOMICS, $17: 5-26$.

Gallant, A., Rossi, P., Tauchen, G., (1993), "Nonlinear Dynamic Structures," ECONOMETRICA, July, Vol. 61, \#4, 871-907.

Gennotte, G. , Leland, H. , (1990), "Market Llquidity, Hedging, and Crashes," AMERICAN ECONOMIC REVIEW, Vol. 88, \# 5, 999-1021.

Grandmont, J., (1992), "Expectations Driven Nonlinear Business Cycles," Cowles Foundation W.P. \#1022.

Grossman, S., (1989), THE INFORMATIONAL ROLE OF PRICES, Cambridge: MIT Press.

Grossman, S., Stiglitz, J., (1980), "On the Impossibility of Informationally Efficient Markets," AMERICAN ECONOMIC REVIEW, 70 (3), June, 393-408.

Harris, M., Raviv, A., (1992), "Differences of Opinion Make a Horse Race," Graduate School of Business, University of Chicago, Graduate School of Management, Northwestern University, REVIEW OF FINANCIAL STUDIES (forthcoming).

Haugen, R. , Talmor, E., Torous, W., (1991), "The Effect of Volatility Changes on the Level of Stock Prices and Subsequent Expected Returns," JOURNAL OF FINANCE, Vol. XIVI, No. 3, pp. 985-1007.

He, H., and Wang, J., (1993), "Differential Information and Dynamic Behavior of Stock Trading Volume," Haas School of Business, University of California, Berkeley and Sloan School of Management, MIT.

Hellwig, M., (1980), "On the Aggregation of Information in Competitive Markets," JOURNAL OF ECONOMIC THEORY, Vol. 22, \#3, June, 477-498.

Hellwig, M., (1982), "Rational Expectations Equilibrium with Conditioning on Past Prices: A Mean-Varlance Example," JOURNAL OF ECONOMIC THEORY, Vol. 26, \#2, Apr 11, 279-312.

Kiefer, N., Ye, Z., An, M., (1993), "A Dynamic Model of Local and Global 
Interactions Among Economic Agents," Department of Economics, Cornell University.

Kurz, M. , (1992), "Asset Prices with Rational Bellefs," Department of Economics, Stanford University.

Lamoureux, C. , Lastrapes, W. , (1990), "Heteroskedasticity in Stock Return Data: Volume versus GARCH Effects," JOURNAL OF FINANCE 45, 221-229.

Lang, L., Litzenberger, R., Madrigal, V., (1992), "Testing Financial Market Equilibrium Under Asymmetric Information," JOURNAL OF POLITICAL ECONOMY, Vol., $100, \quad \# 2,317-348$.

LeBaron, B., (1992), "Persistence of the Dow Jones Index on Rising Volume," SSRI W.P. \#9201, Department of Economics, University of Wisconsin, Madison.

Mankiw, N., (1986), "The Equity Premium and the Concentration of Aggregate Shocks," JOURNAL OF FINANCIAL ECONOMICS, 17, 211-219.

Mansk1, C., and McFadden, D., (1981), STRUCTURAL ANALYSIS OF DISCRETE DATA WITH ECONOMETRIC APPLICATIONS, Cambridge: MIT Press.

Rendleman, R., Jones, C., Latane', H., (1982), "Empirical Anomalies Based on Unexpected Earnings and the Importance of Risk Adjustments," JOURNAL OF FINANCIAL ECONOMICS, November.

Sargent, T., (1993), BOUNDED RATIONALITY IN MACROECONOMICS, Oxford: Clarendon Press.

Stock, J., (1987), "Measuring Business Cycle Time," JOURNAL OF POLITICAL ECONOMY, 95, 1240-1261.

Stock, J., (1988), "Estimating Continuous Time Processes Subject to Time Deformation: An Application to Post War U.S. GNP," JOURNAL OF AMERICAN STATISTICAL ASSOCIATION $83,77-85$.

Varian, H., (1992), "Differences of Opinion," in Newman, P., Milgate, M., Eatwell, J., eds., (1992), THE NEW PALGRAVE DICTIONARY OF MONEY AND FINANCE, London: MacMillan. 
1. W. A. Brock thanks the workshop at the Santa Fe Institute for helpful comments, especially those by Ken Arrow, John Geanokoplous, and Jose' Scheinkman. He is grateful to Ken Arrow for correcting an error in the volume formula. He is also grateful to funding provided by the NSF under Grant Number SES-9122344 and the Vilas Trust. LeBaron thanks the Sloan Foundation and the University of Wisconsin Graduate School for financial support. We thank Steven Durlauf, Nick Kiefer, Wen-Ling Lin, James Stock, three referees, and conference and workshop participants at NBER, SITE, Stern School of Business at New York University, University of Illinois at Champaign-Urbana, and the University of Wisconsin, Madison for helpful comments. None of the above are responsible for errors, opinions, and shortcomings in this paper. This paper was previously titled, "Using Structural Modeling in Building Statistical Models of Volatility and Volume of Stock Market Returns."

2. The compression effect suggests that GARCH type behavior may be coming from the market pricing process itself, rather than the fundamental. This is so because, if the fundamental $\left\{v_{t}\right\}$ is GARCH, a burst of volatility will decay slowly. The compression effect compresses trading volume while fundamental volatility is high. But this is difficult to reconcile with the high contemporaneous correlation between volume and volatility.

3. We digress for a moment to discuss this prediction problem. The dynamics of this prediction problem raise the question whether the system will automatically converge to the Grossman/Stiglitz (1980) equilibrium (cf. Grossman (1989, Chapter 5), Grossman and Stiglitz (1980)) where the fraction of informed traders is set by equating net "profits", or a measure of expected utility, to being informed to "profits" from not being informed.

Brock and Hommes (1994) show the following main result for general prediction systems where cholce over different predictors is governed by profit measures from using each predictor. In other words the result holds for dynamics under adaptive learning over the space of predictors. We state the result here in intultive terms. Assume: (1) nalve, myopic backward looking, or routinized prediction generates a locally unstable equilibrium; (ii) rational expectations or other more sophisticated prediction generates a locally stable equilibrium; (iii) all prediction methods considered by the agents predict the equilibrium next period if the system has converged to the equilibrium; (iv) methods of type (1) cost less per period than methods of type (11). Conclusion: Convergence to equilibrium is a "hairline" event.

An heuristic proof is simple. If convergence is occuring, profits from all methods of prediction that are being used are converging to equality. This is before costs are taken into account. After costs are taken into account only the cheapest prediction methods of type (i) are ultimately being used. But the dynamics are locally unstable when only these methods are used. "QED."

Local instability of equilibrium under short memory backwards looking prediction is associated with high responsiveness of an analogue to a supply curve and low responsiveness of an analogue to a demand curve in the usual cobweb setting. In the information setting to be described below local instability under short memory backwards looking prediction is associated with low risk aversion and low marginal cost of production of signal precision.

The point of raising the issues of instability under adaptive learning over the space of predictors is clear. The theory exposes "far from equilibrium" stabilizing forces that emerge when the gain to using rational prediction covers the costs of using it. However, the theory does not give a strong argument for us to expect the system to settle exactly at a Grossman/Stiglitz equilibrium. The theory suggests that the system will 
occasionally wander out of a neighborhood of the equilibrium, only to be pushed back again when it gets too far away from equllibrium. This movement provides another potential source of persistence of returns volatility and trading volume as well as a potential source of added volatility to returns.

4. The notion of different scales of time is an old one in economics. Stock (1987), (1988) has estimated models where the data are allowed to speak to the speed of passage of the time scale. Writers such as Clark. Tauchen and Pitts, $T$. Andersen, and others cited in the ARCH surveys have stressed the importance of the difference between "economic" time and clock time in finance and how this difference plays a role in generating heteroskedastic effects in returns (cf. Bollerslev, Chou, Kroner (1992), Bollerslev, Engle, and Nelson (1993), Gallant, Rossi, and Tauchen (1993), and their references).

Furthermore the institutions of the market play a role in the production of conditional heteroskedasticity. See the articles in the book edited by Friedman and Rust (1993), especially those by Domowitz and Bollerslev and Domowitz.

Here is another way to think about the idea of two scales of time. The notion of the two scales of time where the fast clock runs fast enough relative to the slow clock for its sample averages to approximately converge, may, perhaps, be thought of as an analytically tractible attempt to apply ideas stimulated by the theory of Kurz (1992) to our problem.

We like to think of Kurz's theory as a metaphor for a situation where the underlying system changes slowly enough so that time averages converge but fast enough so that their convergence on the same data for each agent does not lead to common beliefs, i.e., diversity remains, and, hence, volume will not dry up as in Sargent's (1993) discussion of the no trade theorems and at tempts to break them.

5. We see the potential to formalize the idea of a fast and a slow time scale by using something like the Wald sequential probabllity ratio test (e.g. Basawa and Prakasa Rao (1980)). It is far beyond the scope of this paper to formulize and work out a theory, perhaps along the lines of sequential probability ratio tests, that endogenously produces the two scales of time. Here we simply punt and introduce two scales of time.

There is also a related issue of correlatedness of revision times across agents. We are assuming that all agents entertain switching their information strategies at the same revision times $T, 2 T, \ldots, N T, \ldots$ This is called synchronous adjustment and is present in many economic models, but with little justification. When one starts introducing two time scales of adjustment, this related issue is flushed out into bold relief. Why should traders all entertain switching at the same revision times?.

We could deal with this issue by introducing correlatedness of decisions along the lines of Brock (1993) and allow the data to speak to the degree of correlatedness within the context of the model. Any device that causes the trader heterogeneity to change at a slower time scale than the market time scale appears able to generate temporal persistence in volume and volatility. For example, de Fontnouvelle (1994) extends our model to infinitely lived assets and shows that infinite asset life interacts with trader heterogeneity to generate enough persistence in volume and volatility to be approximately compatible with the data.

It is beyond the scope of this paper to deal with these issues of synchronous adjustment in a serious way. We could assume that across any period $[t, t+1]$ of fast $t$ ime, a fraction " $q$ " of the traders consider belief revision, where $0 \leq q \leq 1$, and a fraction, 1-q, do not entertain revision, and redo all the calculations that appear in this paper. This becomes very messy and is beyond the scope of this paper. Hence, we must punt on the synchrony 
issue and move on.

The introduction of a slower time scale for changing trader characteristics such as their information states will increase the temporal persistence of trading volume which makes the model output more consistent with the data. The presence of a common or correlated signal component in the signal purchased by informed traders can lead to an increase in the volatility of excess returns. Thus the temporal persistence in the fraction of informed traders can lead to temporal persistence in volatility of excess returns.

6. If one introduces a cost externality that leads to a dU(m) that is negative below $m_{*}$, becomes positive on $\left(m_{*}, m^{*}\right)$, and is negative on $\left(m^{*}, \infty\right)$ then

the dynamics become more complicated than fixed points and 2 -cycles as $\rho$ tends to infinity. It is not hard to think of plausible externality structures that lead to current period differential profit functions that rise and then fall in the fraction informed last period. The general point to take away from study of these dynamics is this. An increase in $\rho, 1 . e .$, to borrow Frank Hahn's phrase, "an increase in the dial of rationality," can lead to emergence of complicated dynamics, perhaps even chaos. Since $\rho$ parameterizes a measure of rationality, i.e., intensity of cholce, therefore one could call, perhaps facetiously, the emergence of complex dynamics as $p$ increases, a "rational route to randomness."

7. However, it may be argued that the issues of instability raised by the dynamics treated above are simply artifacts of the naive expectations, $\mathrm{m}^{\mathrm{e}}=\mathrm{m}_{\mathrm{NT}-\mathrm{T}}$. The literature on learning which was surveyed recently by Sargent

(1993) should lead one to be suspicious of results bullt upon naive, short memory, backwards dynamics. Many of the learning dynamics schemes treated in Sargent may lead to convergence to the equilibrium $\bar{m}$, which is the Grossman and Stiglitz (1980) equilibrium. However, if the function $H\left(m_{N T-T}, m_{N T-2 T}, \ldots, m_{N T-L T}\right) \equiv m_{N T-T}$ is replaced by any $H$ that satisfles the "consistency property," $\mathrm{H}(\mathrm{m}, \mathrm{m}, \ldots, \mathrm{m}) \equiv \mathrm{m}$, one may conjecture the following

Proposition: For any consistent $H($.$) , there is a \rho_{c}$ such that for $\rho>\rho_{c^{\prime}}$ the fixed point $m_{\rho}$ for dynamics (2.45) is also a fixed point and is locally unstable for the dynamics

(D. 1) $\mathrm{m}_{\mathrm{NT}}=\tanh \left[(\rho / 2) \mathrm{dU}\left(\mathrm{H}\left(\mathrm{m}_{\mathrm{NT}-\mathrm{T}}, \mathrm{m}_{\mathrm{NT}-2 \mathrm{~T}}, \ldots, \mathrm{m}_{\mathrm{NT}-\mathrm{LT}}\right)\right)\right]$.

Furthermore the fixed point $m_{\rho}$ converges to the Grossman-Stiglitz equilibrium $\overline{\mathrm{m}}$ as $\rho->\infty$.

One can establish this conjecture under modest regularity conditions on $H($. by simple linearization at $m_{\rho}$ and increasing $\rho$ until at least one root of the characteristic equation of the linearization matrix has modulus greater than unity. The conjecture is intuitively obvious because if $\rho$ is very large, dynamics (D.1) approximately sets $m_{N T}$ to the sign of $d U$ which is a discontinuous function. We leave the details to the reader.

8. It might be argued that the above instability was an artifact of lumpiness of the cost function of production of signal precision since there were only two choices: zero precision or precision $\tau_{+}$. To avoid this criticism we sketch below a theory for general cost functions, $c(\tau)$. 
The sketch below will indicate, as was suggested in the introduction, that convergence to Grossman-Stiglitz equilibrium should not be taken for granted. The sketch below gives us the added payoff of a "demand and supply" analytical apparatus for the production of information. Indeed we believe the treatment below is of independent interest.

We describe how a trader chooses signal precision $\tau$. We replace the "lumpy" cost function $c(-1)=0$ for $\tau_{-}=0, c(+1)>0$, for $\tau_{+}>0$, which only allowed production of two levels of $\tau$, by a cost function $c(\tau)$. The cost function $c(\tau)$ is assumed to be twice continuously differentiable, convex, increasing, and $c(0)=0$. A fixed cost component can be added if desired.

Fix all traders" choice of signal precision for the "slab" of fast time contained in $[N T-T, N T]$. Let fraction $n_{j}$ of the traders have precision $\tau_{j}$ and put $\bar{\tau} \equiv \mathrm{h}_{\mathrm{j}} \tau_{\mathrm{j}}$. For a trader with signal precision $\tau$, replace $c(\omega)$ by $c(\tau)$ in Equation (2.37) and follow the same calculations leading to Equation (2.37) to obtain

(2.37b) $U\left(\tau_{j}\right) \equiv(1 / r h)\left[E x^{2}(1+\tau)-E(v-\bar{v}) x\right]-c(\tau), E x^{2}=E[[r h z+v-\bar{v}] /(1+\bar{\tau})]^{2}$,

(2.37c) $E(v-\bar{v}) x=E[(v-\bar{v})([r h z+v-\bar{v}] /(1+\bar{\tau}))=h /(1+\bar{\tau})$,

(2.37d) $E x^{2}=h\left(\left(1+r^{2} h E z^{2}\right)\right] /(1+\bar{\tau})^{2}$.

In order to map this structure into a "supply and demand" analytical apparatus, let $\mathrm{P}=1 /(1+\bar{\tau})^{2}$ play the role of the "price" of information which must be forecasted by agent $j$ before it can intelligently choose $\tau_{j}$. Denote the forecast of $P$ made by trader $f$ by $P_{j}^{e}$. Rewrite $U\left(\tau_{j}\right)$ thus:

(2.37e) $\quad U\left(\tau_{j}\right)=P A\left(1+\tau_{j}\right)-P^{1 / 2} B-c\left(\tau_{j}\right), A \equiv\left(1+r^{2} h E z^{2}\right) / r, B \equiv 1 / r$.

Let $c(\tau)=w \tau+(1 / 2 b) \tau^{2}$ and maximize over $\tau_{j}$ to get the information "supply curve",

(2.37f) $\quad S(P)=c^{\prime-1}(P A)=b(P A-W)$.

Hence if each $\mathrm{j}$ is using a predictor function $\mathrm{H}_{\mathrm{j}}\left(\mathrm{P}_{\mathrm{NT}-\mathrm{T}}\right)$ to predict $\mathrm{P}$ from L-past values $P_{N T-T} \equiv\left(P_{N T-T} \cdots, P_{N T-L T}\right)$, we have

(2.37g) $P \equiv d(Q)=1 /(1+Q)^{2}, Q=D(P) \equiv 1 / P^{1 / 2}-1, Q=\bar{\tau}$, which implies,

(2. 37h) $\left.\quad D\left(P_{t}\right)=\sum \mathrm{h}_{j, N T-T} S\left(\mathrm{H}_{j}\left(P_{N T-T}\right)\right)=\sum \mathrm{h}_{j, N T-T}{ }^{b\left(A H_{j}\right.}\left(P_{N T-T}\right)-W\right)$.

Hence, by suitable interpretation of variables we end up with a dynamical system once we know the $\mathrm{n}_{\mathrm{J}, \mathrm{t}-1}$. But for period [NT-2T, NT-T] agent $\mathrm{J}$ can calculate the actual limiting average trading profits realized during fast time as $T-->\infty$, call this $\pi\left(H_{j}\left(P_{t-2}\right) ; P_{t-1}\right)$, when it chose $\tau_{j}$ based upon (2.37e) with expected $t-1$ price, $P=H_{j}\left(P_{t-2}\right)$, but $P_{t-1}=1 /\left(1+\bar{\tau}_{t-1}\right)^{2}$ actually was realized. Easy calculation shows that $\pi\left(P^{e} ; P\right)$ is given by

( $\pi) \quad \pi\left(P^{e} ; P\right)=P A-P^{1 / 2} B+b\left(P^{e} A-w\right)\left[P A-w-(1 / 2)\left(P^{e} A-w\right)\right]$. 
The results of Brock and Hommes (1994) may now be applied. For example, let there be two predictors available: (i) perfect foresight, $H\left(P_{N T-T}\right)=P_{N T}$ ' avallable at cost $C>0$; naive expectations $H\left(P_{N T-T}\right) \equiv P(N T-T$, avallable at zero cost. Then Brock and Hommes (1994) show: If the dymamics (2.37h) are unstable when everyone uses naive prediction and are stable (in this case instant convergence holds) under perfect foresight, then, there is a critical value of choice intensity $\rho$ such that limit cycles of period greater than two, as well as other "complex" behavior occur. In our context instability of (2.37h) under naive prediction amounts to locating sufficient conditions for the difference equation

$$
\text { (2.37i) } \tau_{t}=b\left[A\left(1 /\left(1+\tau_{t-1}\right)\right)^{2}-w\right] \text {. }
$$

to have an unstable steady state. Obviously this happens if $b$ is large enough. A large b corresponds to a high responsivity of information "supply." We may think of $A$ in (2.37e) as an information "productivity" and/or "level of demand" with the " underlying demand curve" as given in $(2.37 \mathrm{~g})$. We are now ready to think of information equilibrium in terms of the conventional Marshallian scissors of supply and demand. Note that A increases iff the amount of "outside" $r$ isk the system must bear increases. Expected excess profits per share return, $E_{x}=(r h E z) /(1+\bar{\tau})$ increases iff, $P$, the "price" of information and/or rhEz increases.

Since asset price $p$ moves opposite to Ex we may attempt to use our dynamic "information demand and supply" apparatus to interpret phenomena such as (i) blowoffs and crashes; (ii) "bubbles" as in experimental work on asset markets by Vernon Smith and his co-authors, (iii) "stale prices" and "portfolio insurance effects" as in Grossman's book (1989) and his references to Leland, Shiller, and others.

For example, an abrupt rise in information price occurs iff asset price abruptly drops. If learning by doing occurs to lower the marginal cost function of information then volatility around the fundamental can be shown to fall. An increase in portfolio insurance could crudely correspond to a rise in marginal cost of information because traders cannot observe the information generated by the trading in the displaced "real" puts (Grossman (1989)). "Stale prices" could crudely correspond to a rise in the marginal cost function for information.

For example, Madrigal and Scheinkman (1992) put forth a theory that embedded information-strategic market makers into the noisy rational expectations asymmetric information framework to explain the following stylized facts: (1) market breaks seem to be preceded by several more gradual price declines, in response to "bad news", perhaps; (11) volatility increases after the break; (11i) informational role of prices is severely diminished; (iv) bid/ask spreads increase, severe order imbalances appear with market-makers becoming large net buyers of stock.

In Brock and LeBaron (1993), a stripped down version of our apparatus generates through (2.46b) behavior much like (i), (1i). If (iii) is interpreted as less information being produced in our context above, then something like (iii) emerges. More informed traders end up buying lots of stock when a crash occurs, so this is something like (iv).

We believe that it will not be too hard to deal with conditioning upon price so long as we fix the choices $\tau$, at NT-T and simply apply Hellwig's formulas (1980) to calculate the equilibrium at each time in [NT-T,NT] and use this to calculate the analogues to profits, information price, information quantity, etc. above. We have done some preliminary work on this and have 
produced messy but straightforward formulae.

One should eventually use a more appropriate goal measure such as expected utility of profits as in Grossman's book (1989) and to condition on price as well as on signal as in Grossman's book and Hellwig (1980). The case sketched here is good enough to show the spirit of results that we can expect to get.

9. Furthermore one could introduce $K$ components of the fundamental, 1.e. $\mathbf{v}=\sum \mathbf{v}_{\mathbf{k}}$, with a cholce of purchase of a signal $\mathbf{y}_{\mathbf{k}}=\mathbf{v}_{\mathbf{k}}+e_{k}$ for each component $k$. This model should lead to very rich dynamics. One could code the labels of these components into bitstrings as in Arthur (1992) and Arthur etal. (1993) and add J-type cross dependencies for each "slot" of the bitstrings as in Brock (1993). In this way one could develop a parsimoniously parameterized probability structure that generalizes $(2.46 a, b)$ to multiple component bitstring labeling and treat abrupt changes in "feature" selection. We leave this generalization and others to future work. 
APPENDIX ONE: THE SYSTEM EQUATIONS

This Appendix collects all the system equations at one point.

THE SYSTEM EQUATIONS

Here are the system equations.

(S.1) $\operatorname{Max}\left\{E\left[-\exp \left(-r{W_{i t}}\right)\right] \mid I_{1 t}\right\}$, s.t., $p_{t} a_{i t}+b_{1 t}=w_{i t}, w_{1 t}=\left(v_{t}-R p_{t}\right) a_{i t}+R w_{i t}$.

Recall that $v_{t}$ is realized at the end of period $t$, but decisions must be made on the basis of beginning of period $t$ information $I_{i t}$. Recall that under assumptions of conditional normality of $v_{t}$ given $I_{i t}$ ( (cf. LLM (1992, p. 321)) (S.1) this is equivalent to choosing $a_{i t}$ to solve

(S.2) $\operatorname{Max}\left\{E\left[\left(v_{t}-R p_{t}\right) \mid I_{1 t}\right] a_{1 t}-(r / 2) a_{i t}{ }^{2} \operatorname{Var}\left[v_{t} \mid I_{i t}\right]\right.$

Recall that $h \equiv \sigma_{v}^{2} \equiv \operatorname{Var}\left[v_{t} \mid I_{i t}\right]$ denotes the conditional variance of $v_{t} g$ ive the information set $I_{i t} \equiv\left\{I_{t}, y_{i t}\right\}, y_{i t}=v_{t}+e_{i t}$, and $\bar{v}_{t}$ denotes the conditional mean on the publically available and commonly shared information set $I_{t}$. We assume $h$ and $\bar{v}$ are constant through time.

The first order conditions for optimum $a_{i t}$ yield the mean variance demand function,

$$
a_{1 t}=D\left(p_{t} \mid I_{i t}\right) \equiv E\left[\left(v_{t}-R p_{t}\right) \mid I_{1 t}\right] /\left(r \operatorname{Var}\left[v_{t} \mid I_{i t}\right]\right)
$$

Market equilibrium at each time $t$ gives us, assuming no commonly shared signal component, and recalling $\beta \equiv h /\left(h+\sigma_{e}^{2}\right), \tau \equiv h / \sigma_{e}^{2}, \beta_{-}=1, \beta_{+}=h /\left(h+\sigma_{e+}^{2}\right)<1, \tau=0$, $\tau_{+}>0$,

$$
z_{t}=\operatorname{Lim}\left((1 / I) \sum a_{i t}\right)=n_{-t}\left[\bar{v}-R_{p_{t}}\right] /(r h)+n_{+t}\left[\bar{v}+\beta_{+}(v-\bar{v})-R p_{t}\right] /\left(r h\left(1-\beta_{+}\right)\right)
$$

We assume $\beta_{+}$and $\sigma_{e^{+}}{ }^{2}$ are constant through time.

Rewrite (S.4) to obtain (S.5) and (S.6) below,

$$
\begin{aligned}
& v_{t}-R p_{t} \equiv\left[r z_{t} h+\left(v_{t}-\bar{v}_{t}\right)\right] / \bar{\gamma}_{t}^{e}, \\
& \bar{\gamma}_{t}^{e}=\bar{\gamma}_{0}+\left(m_{t}^{e} / 2\right) d \gamma=1+\left(\left(1+m_{t}^{e}\right) / 2\right) \tau_{+} .
\end{aligned}
$$

Recall that $n_{-t}, n_{+t}$, are only allowed to change for $t \varepsilon\{T, 2 T, \ldots, N T, \ldots\}$, $N=1,2, \ldots ; T>1$; but all other variables can change for $t=1,2, \ldots$ This is our way of capturing the notion of slow time and fast time. In the simulations $T$ is a random variable with constant mean bigger than unity. We assign "strengths" to each choice we $\{-1,+1\}$ as follows. The current period profit measure is given by

(S.7) $U(\omega)=\left(1 / r \bar{\gamma}^{e}\right)\left\{\gamma(\omega)\left(r^{2} E\left(z^{2}\right) h\right)+\gamma(\omega)-\bar{\gamma}^{e}\right\}-c(\omega)$. 
Define "strength" $S(\omega)$ by the "distributed lag" difference equation,

$$
s_{t}(\omega)=\left(1 / r \gamma_{t}^{-e}\right)\left\{\gamma(\omega)\left(r^{2} E\left(z^{2}\right) h\right)+\gamma(\omega)-\gamma_{t}^{-e}\right\}-c(\omega)+\eta S_{t-1}(\omega), s_{1}(\omega)=0 \text {. }
$$

At each $t$ ime $t=N T$, we have, letting $d S_{t} \equiv S_{t}(+1)-S_{t}(-1)$, assuming $E\left(z^{2}\right)$ is constant through time,

$$
m_{t}=n_{+t}-n_{-t}=\tanh \left[(\rho / 2) d S_{t}\right] \text {, and for } n=0 \text {, }
$$

$$
m_{t} \equiv_{+t}-n_{-t}=\tanh \left[(\rho / 2)\left\{G\left(m_{t}^{e}\right) \tau_{+}-c_{+}\right\}\right], c_{+} \equiv c(1) \text {, }
$$

$$
G\left(m_{t}^{e}\right) \equiv\left(1 / r \bar{\gamma}_{t}^{-e 2}\right)\left(r^{2} E\left(z^{2}\right) h+1\right) \text {. }
$$

Volume is given by summing the absolute value of the differences in equilibrium demands,

$$
2 r h V=\int I \Delta\left[\left(f_{i}-R p\right) /(1-\beta)\right]\left|d \mu_{2}=\int\right| \Delta\left[\left(f_{i}-R p\right) /(1-\beta)\right] \mid d \mu_{2}=
$$

$\int\left|\Delta[x(1+\tau)]+\Delta[-\varepsilon]+\Delta\left[\tau e_{1}\right]\right| d \mu_{2}$

where the measure $d \mu_{2}$ counts the fraction of traders in period $t-1$, period $t$ who bought (did not buy) the signal in periods $t-1, t$. This calculation depends upon whether $t$ is a revision period or not.

The system evolves as follows. At non revision times $t$, traders who have bought (not bought) the signal recelve a realization (do not receive a realization) at the beginning of period $t$. The fraction $n_{+t}\left(n_{-t}\right)$ of traders who bought the signal (did not buy the signal) was fixed by the discrete choice model, (S.9) at the nearest revision time, call it $N_{1} T$, less than $t$.

The "strengths" that enter (S.9) were calculated based on realizations of actual profits at times in $\left[N_{1} T-T, N_{1} T\right]$. The expectation of the sample analogue of expected profits over $\left[\mathrm{N}_{1} \mathrm{~T}-\mathrm{T}, \mathrm{N}_{1} \mathrm{~T}\right]$ was used to approximate the sample analogue. This approximation makes an error proportional to $1 / T$. We assume $T$ is large enough so that this error is small.

In the actual simulations, we did not make this approximation. We only did it to produce analytical results. At revision times $t=N T$, traders use the discrete choice model (S.9) to choose whether or not to buy the signal. This choice is done independently across traders and independently of their choice at $(\mathrm{N}-1) \mathrm{T}$. Of course a form of dependence can work through the strengths, but there is no other dependence in the choices. At the beginning of revision period NT, after the choice whether or not to buy the signal is made, the system proceeds as described for the nonrevision times.

At the beginning of each period, traders maximize their conditional expected negative exponential utilities of end of period $t$ wealth and send mean variance demands (S.3) to the clearing house, which clears the demands against the realization of supply $z_{t}$. The actual amount paid per share of risky asset is the equilibrium price $p_{t}$ set by the clearing house. The actual number of shares bought or sold by trader $i$ is determined by her demand function evaluated at $p_{t}$. Nature realizes $v_{t}$ and excess profit per share 
$x_{t}=v_{t}-R p_{t}$ and return, $r_{t}=p_{t}-p_{t-1}$ is realized. Perlod $t$ is ended and period $t+1$ begins. The process is repeated at period $t+1$ with a new realization of $v_{t+1}$.

Simulations of versions of the equation system (S.1)-(S.11) are done in Section 3. Those simulations assume an IID process $\left\{v_{t}\right\}$ and produce autocorrelation functions of (1) the equilibrium returns $\mathbf{r}_{t}$ (11) the volatility measure $\left\{r_{t}{ }^{2}\right\}$, (11i) the price volatility measure $\left|R\left(p_{t}-p_{t-1}\right)\right|$, and (iv) the volume measure (S.12). 